OPEN ACCESS

Edited by:

Kang-le Lu,

Jimei University, China

Reviewed by:

Cunxin Sun,

Chinese Academy of Fishery

Sciences, China

M. Virginia Martín,

Instituto Español de Oceanografía

(IEO), Spain

Shuyan Chi,

Guangdong Ocean University, China

${ }^{*}$ Correspondence:

Fei Song

songfei@m.scnu.edu.cn

Specialty section:

This article was submitted to Marine Fisheries, Aquaculture

and Living Resources,

a section of the journal

Frontiers in Marine Science

Received: 14 September 2021

Accepted: 22 October 2021

Published: 30 November 2021

Citation:

Qin $Y, H e C$, Wang W, Yang $P$, Wang J, Qin Q, Mai K and Song F

(2021) Changes in Growth

Performance, Nutrient Metabolism, Antioxidant Defense and Immune

Response After Fishmeal Was

Replaced by Low-Gossypol Cottonseed Meal in Golden Pompano

(Trachinotus ovatus).

Front. Mar. Sci. 8:775575.

doi: 10.3389/fmars.2021.775575

\section{Changes in Growth Performance,} Nutrient Metabolism, Antioxidant Defense and Immune Response After Fishmeal Was Replaced by Low-Gossypol Cottonseed Meal in Golden Pompano (Trachinotus ovatus)

\author{
Yawen Qin'1, Chaoqun He', Wenqiang Wang ${ }^{1}$, Peng Yang ${ }^{1}$, Jun Wang ${ }^{2,3}$, Qinbo Qin ${ }^{4}$, \\ Kangsen Mai ${ }^{1}$ and Fei Song ${ }^{1,2,5 *}$ \\ 1 Guangzhou Key Laboratory of Subtropical Biodiversity and Biomonitoring, Guangdong Provincial Key Laboratory \\ for Healthy and Safe Aquaculture, College of Life Science, Institute of Modern Aquaculture Science and Engineering \\ (IMASE), South China Normal University, Guangzhou, China, ${ }^{2}$ Southern Marine Science and Engineering Guangdong \\ Laboratory, Zhanjiang, China, ${ }^{3}$ Key Laboratory of South China Sea Fishery Resources Exploitation and Utilization, Ministry \\ of Agriculture, South China Sea Fisheries Research Institute, Chinese Academy of Fishery Sciences, Guangzhou, China, \\ ${ }^{4}$ State Key Laboratory of Developmental Biology of Freshwater Fish, College of Life Sciences, Hunan Normal University, \\ Changsha, China, ${ }^{5}$ Southern Marine Science and Engineering Guangdong Laboratory (Zhuhai), Zhuhai, China
}

The present study was designed to elucidate the changing pattern of growth performance, nutrient metabolism, antioxidant defense, and immune response after fishmeal (FM) was replaced by cottonseed meal (CSM). Four isonitrogenous and isolipidic experimental diets (42.5\% crude protein, $14.0 \%$ crude lipid) were formulated to replace 0\% (CSM0 diet), 20\% (CSM20 diet), 40\% (CSM40 diet), and 60\% (CSM60 diet) of the FM protein with CSM. Juvenile golden pompano (Trachinotus ovatus) with an initial body weight of $24.8 \pm 0.02 \mathrm{~g}$ were fed each diet for 6 weeks. The results showed that compared with the control diet, significant differences were not found in the weight gain ratio, specific growth performance, and apparent digestibility of dry matter and protein after 20\% FM was replaced by CSM. Higher CSM replacement markedly decreased the growth performance $(P<0.05)$. Moreover, the CSM20 and CSM40 diets did not depress the feed efficiency ratio and protein efficiency ratio compared with the CSMO diet. Further exploring the physio-biochemical and molecular responses, the present study also showed that dietary 20-40\% CSM replacement had no significant effect on altering the whole body, plasma nutrient contents, free amino acids, or whole-body fatty acid contents. Additionally, the CSM20 diet did not change the mRNA and protein phosphorylation levels of the key enzymes and regulators involved in target of rapamycin (TOR) and amino acid response (AAR) signaling, nutrient metabolism, immune response, and antioxidant defense. Higher CSM inclusion significantly varied the TOR and AAR 
signaling response, nutrient metabolism, immune response, and antioxidant defense. Based on the results, CSM could successfully replace 20\% FM in the formulated diets for golden pompano. This study proposes the optimal inclusion level of CSM in the diet, which has an important significance in optimizing aquafeeds formulation.

Keywords: fishmeal replacement, low-gossypol cottonseed meal, physio-biochemical changes, gene and protein expression, golden pompano (Trachinotus ovatus)

\section{INTRODUCTION}

Although researchers have been devoted to exploring fishmeal (FM) substitution for a few decades, seeking appropriate protein sources to replace FM is still an important mission to achieve sustainable development of aquaculture (Wacyk et al., 2012; Song et al., 2016). In recent decades, a large number of studies have been conducted to assess the efficiency of high-quality plant and animal protein sources in FM replacement (Gatlin et al., 2007; Lu et al., 2015). The results have proved that different protein sources showed diverse substitution efficiencies in different fish species (Song et al., 2014; Wang et al., 2015). In recent years, large quantities of alternative protein sources used in the aquafeeds industry resulted in a competition between humans and animals for food. This phenomenon will directly threaten the entire food security system of the world. Therefore, searching for new nongrain protein sources to replace FM represents a new research topic for aquaculture nutrition studies.

Previously, cottonseed meal (CSM) did not attract much attention as a potential FM substitution in the aquafeeds industry. Currently, an increasing number of researchers have realized that CSM is a special protein source for FM substitution because of its abundance throughout the world and also because it is considered as one of the most important non-grain protein sources for aquafeeds (Lim and Lee, 2009; Ye et al., 2020). The annual cottonseed production in China was more than 3.5 million tons (Hu et al., 2021). Moreover, compared with other alternative protein sources, CSM has relatively higher protein content, wellbalanced amino acid (AA) profile and steady supply is a potential protein source in aquafeeds ( $\mathrm{Li}$ and Robinson, 2006). However, CSM contains a high content of gossypol, and a large quantity of free gossypol in the diet is toxic to fish species, which restricts its utilization in aquafeeds (Zheng et al., 2012). Therefore, CSM in which gossypol was eliminated has become favored because of the improved efficiency of its application in diets.

Previous studies have demonstrated that low-gossypol CSM has different replacement proportions in different species. Research on southern flounder (Paralichthys lethostigma) suggested that low-gossypol CSM could substitute 75\% FM without affecting the growth performance (Sullivan and Reigh, 1995). Anderson et al. (2016) also reported that CSM prepared by solvent extraction could successfully replace dietary $75 \%$ FM in black seabass (Anderson et al., 2016). In Pacific white shrimp (Litopenaeus vannamei), $130 \mathrm{~g} / \mathrm{kg}$ of FM could be replaced by $150 \mathrm{~g} / \mathrm{kg}$ of dephenolized cottonseed protein (Wan et al., 2018). However, a study conducted by $\mathrm{Bu}$ et al. (2017) demonstrated that CSM inclusion over $25.3 \%$ could significantly depress the growth performance, antioxidant capacity, and immune response in the Ussuri catfish Pseudobagrus ussuriensis. All of the literature has elucidated the varying toleration of CSM in different species.

Fishmeal substituted by other protein sources affects nutrient metabolism by regulating nutrient sensing signaling pathways. Our study showed the regulatory role of target of rapamycin (TOR) and amino acid response (AAR) signaling pathways on lipid and glucose metabolism (Song et al., 2016). Studies on the large yellow croaker (Larimichthys crocea) (Wei et al., 2019) and juvenile turbot (Scophthalmus maximus L.) (Wang et al., 2015) also showed that alternative protein meal replacement diets changed growth performance and nutrient metabolism by regulating the TOR signaling pathway. Moreover, FM replacement has also had a great effect on modifying the immune response in fish species (Dossou et al., 2018; Xie et al., 2019). In 2020, Yang et al. reported that largemouth bass fed a poultry by-product meal total substitution diet markedly altered their antioxidant defense and immune response compared with fish fed a FM diet (Yang et al., 2020). Moreover, researchers on red sea bream Pagrus major (Dossou et al., 2018) and hybrid grouper female Epinephelus fuscoguttatus $\times$ male E. lanceolatus (He et al., 2021) provided solid evidence that FM replacement impaired health status by damaging the immune system. Therefore, estimating the changes in nutrient metabolism, immune response, and antioxidant defense status after fish fed alternative protein sources was considered as another important evaluation indicator in assessing the optimal substitution ratio.

Golden pompano (Trachinotus ovatus) is a commercially important marine fish due to its advantage of growing fast, favorable taste, good nutrient profile, and suitability for culture (Tan et al., 2016). In China, it is widely distributed along the coastline of the South China Sea including Guangdong, Fujian, and Hainan provinces. Since it is a carnivorous fish species, golden pompano has a higher requirement for dietary protein (Tang et al., 2013; Xun et al., 2019). Therefore, searching new protein sources to optimize diet formulation has become the key way to achieve healthy and sustainable development of the golden pompano culture industry (Ma et al., 2014). The present study was designed to evaluate the optimal replacement ratio of CSM for FM in golden pompano based on the results of growth performance, physiological and biochemical indexes, metabolism changes, and molecular responses.

\section{MATERIALS AND METHODS}

\section{Ethics Statement}

The experimental procedures strictly complied with the regulations of the University Animal Care and Use Committee 
of the South China Normal University (an approval reference number 1002019-02-0016).

\section{Experimental Diet}

Four isonitrogenous (approximately 42.5\% crude protein) and isolipidic (approximately 14.0\% crude lipid) diets were formulated mainly using FM, CSM, corn-gluten meal, poultry byproduct meal, soybean meal, and peanut meal as the main protein sources, and fish oil, soybean oil, and soybean lecithin as the lipid sources. The FM-based diet was regarded as the control diet (CSM0 diet), and the CSM gradient-substituted FM diet was named the CSM20 diet, CSM40 diet, and CSM60, respectively. Dietary lysine, methionine, and threonine were supplemented to meet the requirements of the golden pompano. The dry ingredients were finely ground into powder through a $320-\mu \mathrm{m}$ screen. Afterward, all compositions used in the diets were individually blended in a mixer and further homogenized after oil and water were added. The pellets were then forced through a pelletizer (F-26, South China University of Technology, Guangzhou, China) and air-dried at $45^{\circ} \mathrm{C}$ to obtain moisture of approximately $10 \%$. All the experimental diets were stored at $-20^{\circ} \mathrm{C}$ until use. The experimental diet formulation is presented in Table $\mathbf{1}$, and the AA composition of the experimental diets is shown in Table 2.

\section{Fish and Experimental Conditions}

Three hundred and sixty golden pompano were purchased from the Dayawan Fish Farm (Guangdong, China). All fish were fed the CSM0 diet two times per day for 2 weeks to apply experimental conditions before the start of the experiment. Afterward, golden pompano were weighed after $24 \mathrm{~h}$ of fasting. Thirst fish with an initial weight of $28.42 \pm 0.02 \mathrm{~g}$ were randomly assigned to sea cages $(1.5 \mathrm{~m} \times 1.5 \mathrm{~m} \times 1.5 \mathrm{~m})$, and each diet was allocated in three replicates. Fish were fed two times (07:00 and 17:00) daily by hand to apparent satiation for 6 weeks. During the trial, the feed consumption, water temperature, and dissolved oxygen were recorded daily. Water temperature ranged from 28 to $30^{\circ} \mathrm{C}$, and dissolved oxygen ranged from 5.0 to $6.0 \mathrm{mg} / \mathrm{L}$.

\section{Sample Collection}

At the end of the feeding trial, the experimental fish were weighed and counted $24 \mathrm{~h}$ after the last feeding, and three fish from each cage were randomly selected for whole body biochemical and fatty acids content measurement. In addition, 12 fish (four per tank) from each treatment were anesthetized with 100 mg/L eugenol (Shanghai Medical Co., Ltd., Shanghai, China). Blood samples were obtained by using the caudal venipuncture method. All samples were put into the anticoagulant tubes and immediately centrifuged at $3,000 \times g$ for $5 \mathrm{~min}$ at $4^{\circ} \mathrm{C}$. The supernatant plasma was placed into liquid nitrogen and then kept at $-80^{\circ} \mathrm{C}$ for analysis. At the same time, the liver, intestine, and kidney were dissected and pooled into RNAase-free tubes (Axygen), frozen in liquid $\mathrm{N}_{2}$, and then stored at $-80^{\circ} \mathrm{C}$ until gene expression analysis.
TABLE 1 | Experimental diets formulations.

\begin{tabular}{|c|c|c|c|c|}
\hline \multirow[t]{2}{*}{ Ingredients } & \multicolumn{4}{|c|}{ Diets (\% dry weight) } \\
\hline & CSMO & CSM20 & CSM40 & CSM60 \\
\hline Fishmeal & 25.00 & 20.00 & 15.00 & 10.00 \\
\hline Cottonseed meal (CSM) & 0.00 & 5.00 & 10.00 & 15.00 \\
\hline Corn gluten meal & 13.00 & 13.00 & 13.00 & 13.00 \\
\hline Poultry by-product meal & 11.00 & 11.00 & 11.00 & 11.00 \\
\hline Soybean meal & 8.50 & 8.50 & 8.50 & 8.50 \\
\hline Peanut meal & 6.50 & 6.50 & 6.50 & 6.50 \\
\hline Wheat meal & 17.50 & 17.50 & 17.50 & 17.50 \\
\hline Fish oil & 1.50 & 2.00 & 2.40 & 2.80 \\
\hline Soybean oil & 5.00 & 5.00 & 5.00 & 5.00 \\
\hline Soybean lecithin & 2.50 & 2.50 & 2.50 & 2.50 \\
\hline Monocalcium phosphate & 1.50 & 1.70 & 1.90 & 2.10 \\
\hline Lysine & 0.28 & 0.45 & 0.60 & 0.75 \\
\hline Methionine & 0.10 & 0.15 & 0.20 & 0.25 \\
\hline Threonine & 0.01 & 0.03 & 0.05 & 0.07 \\
\hline Squid paste & 1.50 & 1.50 & 1.50 & 1.50 \\
\hline Mineral premix ${ }^{a}$ & 1.50 & 1.50 & 1.50 & 1.50 \\
\hline Vitamin premix ${ }^{b}$ & 0.50 & 0.50 & 0.50 & 0.50 \\
\hline Chromium trioxide & 0.10 & 0.10 & 0.10 & 0.10 \\
\hline Lutein & 0.10 & 0.10 & 0.10 & 0.10 \\
\hline Antioxidant & 0.05 & 0.05 & 0.05 & 0.05 \\
\hline Mold inhibitor & 0.10 & 0.10 & 0.10 & 0.10 \\
\hline Cellulose & 3.76 & 2.82 & 2.00 & 1.18 \\
\hline
\end{tabular}

Proximate composition

\section{$\mathrm{DM}(\%)$}

Crude protein (\%)

90.24

42.42

\subsection{8}

89.91

90.12

Crude lipid (\%)

14.00

42.51

42.58

42.66

a Mineral premix (mg/kg diet): NaF, $2 \mathrm{mg} ; \mathrm{Kl}, 0.8 \mathrm{mg} ; \mathrm{CoCl}_{2} \cdot 6 \mathrm{H}_{2} \mathrm{O}(10 \mathrm{~g} / \mathrm{kg}), 50 \mathrm{mg}$; $\mathrm{CuSO}_{4} \cdot 5 \mathrm{H}_{2} \mathrm{O}, 10 \mathrm{mg} ; \mathrm{FeSO}_{4} \cdot \mathrm{H}_{2} \mathrm{O}, 80 \mathrm{mg} ; \mathrm{ZnSO}_{4} \cdot \mathrm{H}_{2} \mathrm{O}, 50 \mathrm{mg} ; \mathrm{MnSO}_{4} \cdot \mathrm{H}_{2} \mathrm{O}, 60$ mg; $\mathrm{MgSO}_{4} \cdot 7 \mathrm{H}_{2} \mathrm{O}, 1,200 \mathrm{mg} ; \mathrm{Ca}\left(\mathrm{H}_{2} \mathrm{PO}_{4}\right)_{2} \cdot \mathrm{H}_{2} \mathrm{O}$, 3,000 mg; $\mathrm{NaCl}, 100$ mg; zeolite, $15,447 \mathrm{mg}$.

${ }^{b}$ Vitamin premix (mg/kg diet): thiamin, $25 \mathrm{mg}$; riboflavin, $45 \mathrm{mg}$; pyridoxine $\mathrm{HCl}, 20$ mg; vitamin B12, 0.1 mg; vitamin K3, 10 mg; inositol, 800 mg; pantothenic acid, 60 mg; niacin acid, 200 mg; folic acid, 20 mg; biotin, 1.20 mg; retinal acetate, 32 mg; cholecalciferol, $5 \mathrm{mg}$; $\alpha$-to-copherol, $120 \mathrm{mg}$; ascorbic acid, 2,000 mg; choline chloride, 2,500 mg; ethoxyquin $150 \mathrm{mg}$; wheat middling, 14,012 mg.

\section{Digestibility Trial}

A digestibility trial was conducted during the feeding trial period. Chromium trioxide (0.1\%) (99.9\%, Sigma Co., Ltd., MO, United States) was used as the indicator in the diets. The collected feces were dried for $6 \mathrm{~h}$ at $65^{\circ} \mathrm{C}$ and stored at $-20^{\circ} \mathrm{C}$ until analysis. The content of chromium trioxide in the diet and feces was measured by perchloric acid digestion and determined by inductively coupled plasma-atomic emission spectrophotometry (ICP-OES, VISTA- MPX) (Bian et al., 2017).

\section{Proximate Composition Analysis}

A proximate composition analysis was conducted following previous studies (Cai et al., 2020). Moisture was measured by drying samples to a constant weight in an oven at $105^{\circ} \mathrm{C}$. Crude protein was detected using the Dumas nitrogen determination apparatus (DT autosampler, Europe Gerhardt 
TABLE 2 | The essential amino acids composition of the experimental diets.

\begin{tabular}{lcccc}
\hline Amino acids & \multicolumn{5}{l}{ Diets (\% dry weight) } & \\
\cline { 2 - 5 } & CSM0 & CSM20 & CSM40 & CSM60 \\
\hline Lys & 1.90 & 1.92 & 1.92 & 1.91 \\
Met & 0.70 & 0.70 & 0.70 & 0.70 \\
Thr & 0.88 & 0.88 & 0.88 & 0.88 \\
Arg & 1.29 & 1.51 & 1.73 & 1.95 \\
His & 0.47 & 0.49 & 0.51 & 0.53 \\
Ile & 0.84 & 0.81 & 0.79 & 0.77 \\
Leu & 1.51 & 1.48 & 1.45 & 1.42 \\
Phe & 0.89 & 0.94 & 0.99 & 1.04 \\
Val & 1.02 & 1.01 & 0.99 & 0.97 \\
Cys & 0.15 & 0.18 & 0.20 & 0.23 \\
Tyr & 0.66 & 0.65 & 0.65 & 0.64 \\
\hline
\end{tabular}

Company, Germany). The Soxtec ${ }^{\mathrm{TM}} 2055$ extraction by using petroleum ether (B.P. $30-60^{\circ} \mathrm{C}$ for $3 \mathrm{~h}$ ) was used for crude lipid determination. Ash was determined by combustion in a muffle furnace (FO610C, Yamato Scientific Co., Ltd., Tokyo, Japan) to a constant weight at $550^{\circ} \mathrm{C}$. The ingredients and diets were dried by a freezer dryer (ALPHA1-2 LD plus, Christ Co., Ltd., Germany) to determine the AA concentrations. After digestion with $6 \mathrm{M}$ $\mathrm{HCl}$ for $22 \mathrm{~h}$, all the AA compositions in the ingredients and diets were measured by an L-8900 AA analyzer (Hitachi, Japan).

\section{Analysis of Free Amino Acid and Fatty Acids}

The free AA composition in plasma was analyzed using an auto AA analyzer (LA8080; Hitachi, Tokyo, Japan). The details were described by Wang et al. (2020). Briefly, $1.2 \mathrm{ml}$ of $10 \%$ sulfosalicylic acid solution and $400 \mu \mathrm{l}$ of each plasma sample were mixed well. After incubation for $5 \mathrm{~min}$ at $4^{\circ} \mathrm{C}$, all the samples were centrifuged at $13,000 \mathrm{rpm}$ for $15 \mathrm{~min}$ at $4^{\circ} \mathrm{C}$. Supernatants were filtered through $0.22-\mu \mathrm{m}$ filters for free AA concentration analysis. The fatty acid composition of the whole body was measured following the methodology of Mourente et al. (1999) with some modifications (Xu et al., 2010).

\section{Determination of Plasma and Liver Biochemical Parameters}

The measurement of plasma and liver biochemical parameters was followed by Yang et al. (2020). Around $200 \mu$ l of each plasma sample was used for this detection. The concentrations of total glucose (GLU), total protein (TP), triglycerides (TG), total cholesterol (CHOL), high-density lipoprotein (HDL), low-density lipoprotein (LDL), aspartate aminotransferase (AST), alanine aminotransferase (ALT), alkaline phosphatase (ALP), lactate dehydrogenase (LDH), and creatine kinase (CK) in plasma were measured by using an Automatic Biochemical Analyzer (Sysmex Corporation, CHEMIX800, Kobe, Japan).

\section{RNA Extraction and Quantitative Real-Time PCR}

Total RNA was extracted by using the TRIzol method (Vazyme Biotech Co., Ltd., China) following the manufacturer's instructions. After RNA eluted in diethyl pyrocarbonate (DEPC)treated water, the quantity and quality of isolated RNA were measured using NanoDrop 2000 spectrophotometer (Thermo, NanoDrop Technologies, United States) and electrophoresis in a $1.2 \%$ agarose gel, respectively. One microgram of RNA was transcribed into cDNA by using the Prime Script RT reagent Kit (Vazyme Biotech Co., Ltd., China). The cDNA templates were diluted to $100 \mathrm{ng} / \mu \mathrm{L}$ with $\mathrm{DEPC}$ water before use for quantitative real-time PCR analysis.

Primers used in this study are shown in Table 3. Targeted gene expression levels were determined by quantitative RT-PCR carried out on a CFX96 real-time PCR machine (CFX96, BIORAD, United States) using Hiff ${ }^{\circledast}$ qPCR SYBR Green Master Mix (Yeasen, Shanghai, China). The thermal cycle program of qRTPCR was as follows: $95^{\circ} \mathrm{C}$ for $2 \mathrm{~min}$, then 40 cycles at $95^{\circ} \mathrm{C}$ for $15 \mathrm{~s}, 58^{\circ} \mathrm{C}-60^{\circ} \mathrm{C}$ for $30 \mathrm{~s}$, and $72^{\circ} \mathrm{C}$ for $20 \mathrm{~s}$. Melting curve analysis after the end of each PCR was executed to confirm that only one single product was present in these reactions. Relative expression levels of target genes were calculated using the comparative CT method ( $2^{-\Delta \Delta C t}$ method). Relatively gene expression is represented as the fold change relative to the control group (Zhou et al., 2019).

\section{Western Blotting}

The protein expression level of total TOR, phospho-TOR, total ribosomal protein S6 (S6), phospho-S6, totalith phosphatase inhibitor and protease cocktails (Bimake, TX, United States) for $1 \mathrm{~h}$. The protein concentrations of all the samples were measured by using the BCA protein assay kit (Beyotime, Biotechnology, China). Samples were separated by sodium dodecyl sulfate-polyacrylamide gels (SDS-PAGE) and transferred to $0.45-\mu \mathrm{m}$ PVDF membranes (Millipore). After blocking with $5 \%$ non-fat milk in TBST buffer $(20 \mathrm{mM}$ Tris- $\mathrm{HCl}$, $500 \mathrm{mM} \mathrm{NaCl}, 0.1 \%$ Tween-20) for $1 \mathrm{~h}$, the membranes were incubated with primary antibody (Cell Signaling Technology, MA, United States and Santa Cruz Biotechnology Inc., TX, United States) overnight at $4^{\circ} \mathrm{C}$. All of the primary antibodies have been shown to be conserved in fish species and could be used successfully (Song et al., 2016; Wang et al., 2020). Then, the membranes were incubated in horseradish peroxidase-labeled secondary antibodies for $1 \mathrm{~h}$ and visualized using ECL reagents (Beyotime Biotechnology, China). The Western blot result bands were quantified with $\mathrm{NIH}$ Image 1.63 software. All the antibodies in the present study were confirmed to be conserved and successfully used in fish species.

\section{Calculations and Data Analysis}

Weight gain rate $(\mathrm{WGR}, \%)=100 \times\left(\mathrm{W}_{t}-\mathrm{W}_{0}\right) / \mathrm{W}_{0}$;

Specific growth rate $(\mathrm{SGR}, \% /$ day $)=100 \times\left(\mathrm{Ln} \mathrm{W}_{t}-\mathrm{Ln} \mathrm{W}_{0}\right) / \mathrm{t}$; Feed efficiency ratio $($ FER $)=\left(\mathrm{W}_{t}-\mathrm{W}_{0}\right) /($ dry feed intake $)$; 
TABLE 3 | The sequences of the primers used in the RT-PCR.

\begin{tabular}{|c|c|c|}
\hline $\begin{array}{l}\text { Target } \\
\text { gene }\end{array}$ & Forward sequence $\left(5^{\prime}-3^{\prime}\right)$ & Reverse sequence $\left(5^{\prime}-3^{\prime}\right)$ \\
\hline TOR & $\begin{array}{l}\text { GGGTCTTATGAGCCAGTGC } \\
\text { CAGG }\end{array}$ & $\begin{array}{l}\text { CTTCAGGGTTGTCAGCGGA } \\
\text { TTGT }\end{array}$ \\
\hline S6 & GCACTGTCCCTCGCCGTCTT & CTGGGCTTCTTGCCTTCTाT \\
\hline 4EBP1 & ACACCCCAGCAGGAACTIT & GTGACCATCAACGACGCAG \\
\hline $\mathrm{elF} 2 \alpha$ & $\begin{array}{l}\text { TGTATTCCAGCACCTC } \\
\text { AGCC }\end{array}$ & $\begin{array}{l}\text { CGTGGTCGTCATCCGA } \\
\text { GTAGA }\end{array}$ \\
\hline ATF4 & CTGCGTCACCССTCAАCTCC & САТТСGСТССАТССАСААСС \\
\hline $\mathrm{CHOP}$ & $\begin{array}{l}\text { CGGAGTTCTGGATGTT } \\
\text { TTGGA }\end{array}$ & $\begin{array}{l}\text { AGGAGGAGGAAGAGGAG } \\
\text { GATGA }\end{array}$ \\
\hline REDD1 & $\begin{array}{l}\text { AGCCAAAGACTCAGAAT } \\
\text { GCG }\end{array}$ & $\begin{array}{l}\text { TGAAAGGTGGGGACAA } \\
\text { GGTA }\end{array}$ \\
\hline FAS & GATGGATACAAAGAGCAAGG & GTGGAGCCGATAAGAAGA \\
\hline ACC & GTTGTCAATCCCAGCCGATC & ATCCACAATGTAGGCCCCAA \\
\hline AGPAT3 & CTTCCTGTITGGGCCACTC & GTCGCCATAACTTGAGCCTG \\
\hline FAD2a & GAACAATCCCACTTCAACG & AGGAATCCCATACTTCTCACA \\
\hline elov15 & $\begin{array}{l}\text { TACATGGTCACGCTCATTA } \\
\text { TCC }\end{array}$ & $\begin{array}{l}\text { CCGTTCTGATGCTCCTTC } \\
\text { ПTA }\end{array}$ \\
\hline SREBP1 & GAGCCAAGACAGAGGAGTGT & GTCCTCTTGTCTCCCAGCTा \\
\hline LPL & TाGTCCTTCCTCGTCACCA & AAGACAGCATCCTCTCCACC \\
\hline HSL & TCATACCTCCACACCAACCC & GTCTCGCAGTITCTTGGCAA \\
\hline CPT1 & СTTAGCCAAGCCCTTCATC & CACGGTTACCTGTTCCCTCT \\
\hline PPAR $\gamma$ & TCAGGGTTCACTATGGCGT & CTGGAAGCGACAGTATTGGC \\
\hline $\operatorname{PPAR} \alpha$ & AATCTCAGCGTGTCGTCTT & GGAAATGCTTCGGATACTTG \\
\hline FABP1 & CCAAGGACATCAAGCCAATTAC & TGGTGATTTCAGCCTCCTTAC \\
\hline APRO & AAAAGCCACAAGACGAA & GAAGCAGCAAAAGGCA \\
\hline B100 & AGCA & GAGC \\
\hline g6pdh & CTGTGGCAAAAGTTGGTGTG & CCTGATGATGTGAGGGATGA \\
\hline HK & ССTTCCTCGTCTITGTCATT & TGTCCGTCTCATCCTGGTG \\
\hline PK & TाTGCCAGTTCATCCGCT & CCATCACGCCATCGCTCT \\
\hline pfk-1 & TGGGTGGGACCGTGATT & AGGTTGGTGATGCCTTCTT \\
\hline pepck & $\begin{array}{l}\text { TGGAGTGTTTGTTGGAG } \\
\text { CAG }\end{array}$ & $\begin{array}{l}\text { CGAAGTTGTAGCCGAA } \\
\text { GAAG }\end{array}$ \\
\hline glut2 & TCCTGTTGCTGTGCTGCTT & GTITCCGTCCCTTGCG \\
\hline glut4 & AATGGCTGTGGCTGGCTT & AGGTIITCCCCGTGTTCT \\
\hline IL-10 & $\begin{array}{l}\text { CTCCAGACAGAAGACTCC } \\
\text { AGCA }\end{array}$ & $\begin{array}{l}\text { GGAATCCCTCCACAAAA } \\
\text { CGAC }\end{array}$ \\
\hline IL-8 & $\begin{array}{l}\text { TGCATCACCACGGTG } \\
\text { AAAAA }\end{array}$ & $\begin{array}{l}\text { GCATCAGGGTCCAGACA } \\
\text { AATC }\end{array}$ \\
\hline TGF- $\beta 1$ & GAGATACGGAAAAGAGTGGGG & TGACAAAGCGGGAAGCAAG \\
\hline C4 & $\begin{array}{l}\text { TGGAGAAAAAGTTAAAG } \\
\text { GGGC }\end{array}$ & $\begin{array}{l}\text { CAGGAAGGAAGTATGAGC } \\
\text { GAGT }\end{array}$ \\
\hline C-lyz & $\begin{array}{l}\text { GGAGTCTGGTGTTCTGCTC } \\
\text { TाTG }\end{array}$ & $\begin{array}{l}\text { GGTGGCTCTAGTGTTGTAG } \\
\text { TTCG }\end{array}$ \\
\hline HSP70 & $\begin{array}{l}\text { TTGAGGAGGCTGCGCACAGCT } \\
\text { TGTG }\end{array}$ & $\begin{array}{l}\text { ACGTCCAGCAGCAGCAGG } \\
\text { TCCT }\end{array}$ \\
\hline HSP9O & $\begin{array}{l}\text { GATGAAAAGGCGTTTGAGAAAA } \\
\text { TGAT }\end{array}$ & $\begin{array}{l}\text { TGTTGCAGGCTTGATGTT } \\
\text { GAGTACAC }\end{array}$ \\
\hline ІкB- $\alpha$ & $\begin{array}{l}\text { GCTGGTGAACGGGT } \\
\text { TTGAG }\end{array}$ & $\begin{array}{l}\text { GAGGAAGGGGTGATTG } \\
\text { TGTGA }\end{array}$ \\
\hline MnSOD & AGCCAGCCTCAGCCAAACT & GGCGGTGACATCTCCCTIT \\
\hline CAT & AGTITACACCGAGGAGGGC & TGTGGGTTTGGGGATTGC \\
\hline Nrf2 & $\begin{array}{l}\text { TTGCCTGGACACAACTGCTG } \\
\text { TTAC }\end{array}$ & $\begin{array}{l}\text { TCTGTGACGGTGGCAGT } \\
\text { GGAC }\end{array}$ \\
\hline Keap1 & $\begin{array}{l}\text { CAGATAGACAGCGTGGTGA } \\
\text { AGGC }\end{array}$ & $\begin{array}{l}\text { GACAGTGAGACAGGTTGAA } \\
\text { GAACTCC }\end{array}$ \\
\hline $\mathrm{HO}-1$ & $\begin{array}{l}\text { AGAAGATTCAGACAGCAGCAG } \\
\text { AACAG }\end{array}$ & $\begin{array}{l}\text { TCATACAGCGAGCACAGGA } \\
\text { GGAG }\end{array}$ \\
\hline$\beta$-actin & TACGAGCTGCCTGACGGACA & GCTGTGATCTCCTTCTGC \\
\hline
\end{tabular}

Protein efficiency ratio $(\mathrm{PER})=\left(\mathrm{W}_{t}-\mathrm{W}_{0}\right) /($ dry feed intake $\times$ protein percent in dry diet);

Feed intake $($ FI, \% body weight $/ \mathrm{d})=100 \times$ feed fed/[days $\times$ (initial weight + final weight $) / 2]$

Protein productive value $(\mathrm{PPV}, \%)=\left(\mathrm{W}_{t} \times \mathrm{P}_{t}-\right.$ $\left.\mathrm{W}_{0} \times \mathrm{P}_{0}\right) /\left(\mathrm{W}_{d} \times \mathrm{P}_{d}\right) \times 100$;

Survival rate $(\mathrm{SR}, \%)=100 \times$ final amount of fish/initial amount of fish;

Protein retention $(\mathrm{PR}, \%)=100 \times($ final body weight $\times$ final carcass protein content - initial body weight $\times$ initial carcass protein content)/protein intake;

Dry matter apparent digestibility (AD dry matter, $\%)=[1-$ chromium trioxide in feed/chromium trioxide in feces $] \times 100 \%$;

Apparent digestibility of protein in feed (AD for protein, $\%)=[1-($ chromium trioxide in diet $/$ chromium trioxide in feces) (nutrient in feces/nutrient in diet)] $\times 100 \%$.

$\mathrm{W}_{0}, \mathrm{~W}_{t}$, and $\mathrm{W}_{d}$ represent the body weight $(\mathrm{g})$ of initial fish, final fish, and diet dry weight, respectively, $\mathrm{P}_{0}, \mathrm{P}_{t}$, and $\mathrm{P}_{d}$ represent the body protein content (\%) of initial fish, final fish, and diet dry matter, respectively, while $t$ represents the rearing days of this experiment.

Data were expressed as means \pm SEM. All statistical evaluations were analyzed using one-way analysis of variance (ANOVA) followed by Tukey's multiple range test with SPSS 19.0 software. Prior to the statistical tests, data in the present study were examined for homogeneity of variances. In cases in which data were non-parametric or not homoscedastic, data transformations (such as logarithms, square roots, and reciprocals) were used to meet ANOVA criteria. Differences were considered significant when $P<0.05$.

\section{RESULTS}

\section{Growth Performance, Apparent Digestibility, and Whole Body Proximate Composition}

Growth performances were shown in Table 4. Compared with the CSM0 diet, the CSM20 diet did not significantly decrease the FBW, WGR, SGR, FER, PER, PR, AD (dry matter), or $\mathrm{AD}$ (protein) $(P>0.05)$. However, the CSM40 and CSM60 diet groups had markedly lower levels of FBW, WGR, SGR, PPV, PR, AD (dry matter), and AD (protein) compared with the control diet group $(P<0.05)$. Moreover, compared with the control diet, the CSM20 and CSM40 diets did not depress the FER, PER, or whole-body proximate crude fat composition $(P>0.05)$. The CSM60 diet significantly reduced the FER, PER, and crude fat composition compared with the CSM0 diet $(P<0.05)$. No significant differences in FI, SR, whole-body moisture, crude protein, or ash were found among the different treatments $(P>0.05)$.

\section{Plasma Biochemical Index}

At the end of the feeding trial, the effects of replacing FM with CSM on the plasma nutrient content were shown in Table 5. 


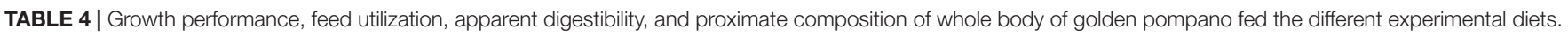

\begin{tabular}{|c|c|c|c|c|c|}
\hline & \multicolumn{4}{|c|}{ Diet groups } & \multirow[t]{2}{*}{$P$-value } \\
\hline & CSMO & CSM20 & CSM40 & CSM60 & \\
\hline IBW (g) & $28.38 \pm 0.04$ & $28.40 \pm 0.05$ & $28.45 \pm 0.02$ & $28.44 \pm 0.06$ & 0.70 \\
\hline FBW (g) & $92.67 \pm 1.74^{a}$ & $86.70 \pm 1.79^{a b}$ & $85.41 \pm 0.79^{b c}$ & $79.46 \pm 1.20^{\mathrm{C}}$ & 0.00 \\
\hline WGR (\%) & $226.56 \pm 6.61^{a}$ & $205.32 \pm 6.82^{a b}$ & $195.70 \pm 6.40^{b}$ & $179.44 \pm 4.40^{b}$ & 0.04 \\
\hline $\operatorname{SGR}(\% / d)$ & $2.82 \pm 0.05^{a}$ & $2.66 \pm 0.06^{a b}$ & $2.58 \pm 0.05^{b}$ & $2.45 \pm 0.04^{b}$ & 0.04 \\
\hline FER & $0.95 \pm 0.02^{a}$ & $0.90 \pm 0.02^{a b}$ & $0.87 \pm 0.04^{a b}$ & $0.82 \pm 0.02^{b}$ & 0.03 \\
\hline PER & $2.24 \pm 0.043^{a}$ & $2.11 \pm 0.033^{a b}$ & $2.09 \pm 0.047^{a b}$ & $1.93 \pm 0.050^{b}$ & 0.007 \\
\hline FI (\% body weight/d) & $2.96 \pm 0.025$ & $2.98 \pm 0.03$ & $3.02 \pm 0.08$ & $3.06 \pm 0.04$ & 0.08 \\
\hline PPV (\%) & $43.41 \pm 0.10^{\mathrm{a}}$ & $41.45 \pm 0.01^{b}$ & $40.33 \pm 0.03^{c}$ & $38.19 \pm 0.23^{d}$ & 0.00 \\
\hline SR (\%) & $99.0 \pm 1.00$ & $99.0 \pm 1.00$ & $100.0 \pm 0.00$ & $99.0 \pm 1.00$ & 0.36 \\
\hline PR (\%) & $39.68 \pm 0.74^{a}$ & $37.80 \pm 0.49^{a b}$ & $36.06 \pm 0.33^{b c}$ & $35.23 \pm 0.28^{c}$ & 0.01 \\
\hline AD (Dry mater) & $84.47 \pm 2.78^{a}$ & $78.09 \pm 3.87^{a b}$ & $55.07 \pm 8.78^{\mathrm{bc}}$ & $47.80 \pm 6.24^{c}$ & 0.06 \\
\hline AD (Protein) & $92.35 \pm 1.48^{\mathrm{a}}$ & $88.94 \pm 1.98^{a b}$ & $79.53 \pm 4.17^{b}$ & $76.80 \pm 2.59^{b}$ & 0.01 \\
\hline \multicolumn{6}{|c|}{ Proximate composition of whole body (wet weight\%) } \\
\hline Moisture & $62.3 \pm 0.54$ & $62.7 \pm 0.85$ & $63.2 \pm 0.24$ & $63.5 \pm 0.12$ & 0.44 \\
\hline $\mathrm{CP}$ & $18.2 \pm 0.076$ & $18.2 \pm 0.21$ & $17.6 \pm 0.11$ & $18.0 \pm 0.19$ & 0.06 \\
\hline CF & $15.1 \pm 0.37^{\mathrm{a}}$ & $14.5 \pm 0.17^{a b}$ & $14.3 \pm 0.05^{a b}$ & $13.6 \pm 0.17^{b}$ & 0.01 \\
\hline Ash & $3.99 \pm 0.086$ & $3.93 \pm 0.10$ & $4.08 \pm 0.10$ & $3.98 \pm 0.17$ & 0.84 \\
\hline
\end{tabular}

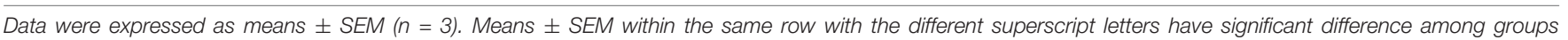
$(P<0.05)$.

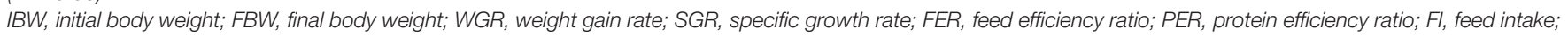

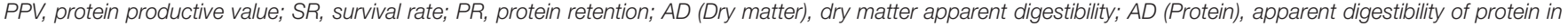
feed; CP, crude protein; CF, crude fat.

TABLE 5 | Plasma biochemical index of golden pompano fed different experimental diets for 6 weeks.

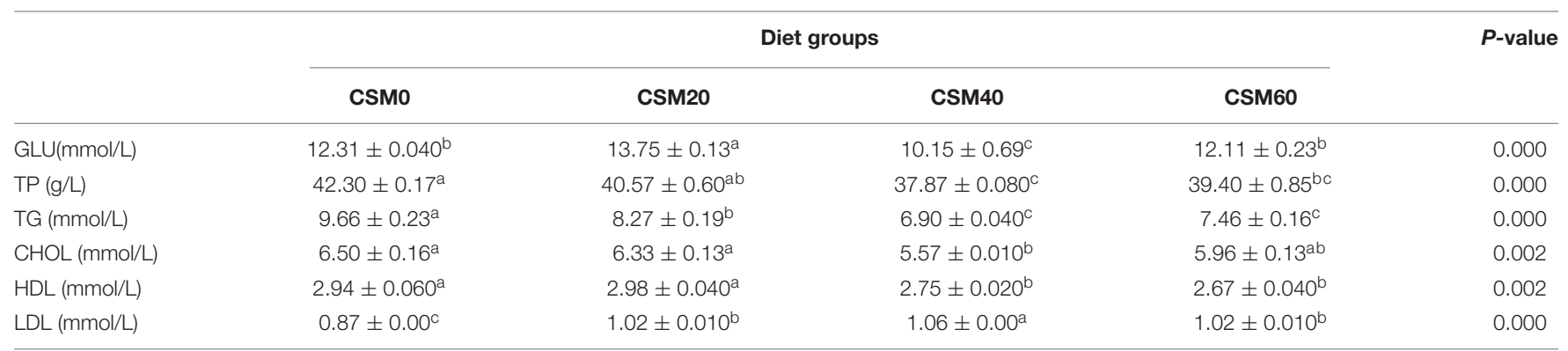

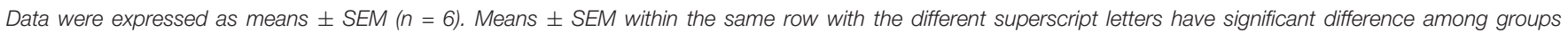
$(P<0.05)$.

GLU, total glucose; TP, total protein; TG, triglycerides; CHOL, total cholesterol; HDL, high-density lipoprotein; LDL, low density lipoprotein.

The CSM20 diet had no effect on decreasing the content of plasma TP, CHOL, and HDL compared with the CSM0 diet $(P>0.05)$. In contrast, the CSM20 diet elevated plasma GLU compared with the control diet $(P<0.05)$. However, the CSM40 diet group had lower plasma level of GLU, TP, TG, CHOL, and HLD, and the CSM60 diet group had lower plasma level of TP, TG, and HLD compared with the CSM0 diet group $(P<0.05)$.

\section{The Plasma and Liver Enzyme Activities}

The activities of markers indicating liver damage in plasma and liver were shown in Table 6. The activities of AST, ALT, ALP, and $\mathrm{LDH}$ were markedly increased in the liver with increasing $\mathrm{CSM}$ replacement in diets among groups $(P<0.05)$. Only
20\% CSM replacing FM could markedly improve the activity of these four enzymes $(P<0.05)$. In plasma, the CSM20 diet group had no significant difference in the enzyme activities of ALT and ALP in the plasma compared with the CSM0 diet group $(P>0.05)$. Moreover, compared with the CSM0 diet, the CSM40 and CSM60 diets significantly elevated the activity of AST and inhibited the activity of $\operatorname{ALP}(P<0.05)$. There was no significant difference in $\mathrm{LDH}$ activity among the different groups $(P>0.05)$.

\section{Plasma Free Amino Acid Profile}

The free AA profiles in plasma after fish were fed with different diets were shown in Table 7. Both the CSM20 and CSM40 diets had no effect on altering all the individual 
TABLE 6 | The enzymes activities of golden pompano feed different experimental diets in the plasma and liver.

\begin{tabular}{|c|c|c|c|c|c|}
\hline & \multicolumn{4}{|c|}{ Diet groups } & \multirow[t]{2}{*}{$P$-value } \\
\hline & CSMO & CSM20 & CSM40 & CSM60 & \\
\hline \multicolumn{6}{|l|}{ Liver } \\
\hline $\mathrm{AST}(\mathrm{U} / \mathrm{g})$ & $2039.95 \pm 6.55^{d}$ & $2420.81 \pm 0.89^{c}$ & $2587.73 \pm 3.51^{b}$ & $2728.93 \pm 3.24^{a}$ & 0.00 \\
\hline $\mathrm{ALT}(\mathrm{U} / \mathrm{g})$ & $297.87 \pm 1.55^{d}$ & $362.26 \pm 1.48^{\mathrm{c}}$ & $423.39 \pm 1.53^{a}$ & $394.50 \pm 0.87^{b}$ & 0.00 \\
\hline$A L P(U / g)$ & $58.87 \pm 0.22^{\mathrm{c}}$ & $65.22 \pm 0.30^{\mathrm{b}}$ & $72.49 \pm 0.30^{a}$ & $72.44 \pm 0.50^{a}$ & 0.00 \\
\hline $\mathrm{LDH}(\mathrm{U} / \mathrm{g})$ & $75.46 \pm 0.44^{b}$ & $96.94 \pm 0.51^{a}$ & $104.84 \pm 4.3^{a}$ & $97.461 \pm 0.29^{a}$ & 0.00 \\
\hline \multicolumn{6}{|l|}{ Plasma } \\
\hline AST (U/L) & $46.00 \pm 0.00^{c}$ & $40.67 \pm 0.33^{d}$ & $54.67 \pm 0.33^{a}$ & $50.00 \pm 0.00^{b}$ & 0.00 \\
\hline ALT (U/L) & $4.67 \pm 0.33^{b}$ & $5.00 \pm 0.00^{b}$ & $5.00 \pm 0.00^{b}$ & $7.00 \pm 0.00^{\mathrm{a}}$ & 0.00 \\
\hline ALP (U/L) & $51.67 \pm 0.33^{a}$ & $51.33 \pm 0.33^{a}$ & $46.00 \pm 0.00^{c}$ & $49.33 \pm 0.67^{b}$ & 0.00 \\
\hline LDH (U/L) & $187.67 \pm 17.23$ & $164.00 \pm 1.15$ & $234.33 \pm 30.67$ & $189.33 \pm 6.84$ & 0.12 \\
\hline
\end{tabular}

Data were expressed as means \pm SEM $(n=6)$.

Means \pm SEM within the same row with the different superscript letters have significant difference among groups $(P<0.05)$.

AST, aspartate aminotransferase; ALT, alanine aminotransferase; ALP, alkaline phosphatase; $L D H$, lactate dehydrogenase.

TABLE 7 | The free amino acids concentration after golden pompano feed with different diets in the plasma ( $\mu \mathrm{g} / \mathrm{ml})$.

\begin{tabular}{|c|c|c|c|c|c|}
\hline & \multicolumn{4}{|c|}{ Diet groups } & \multirow[t]{2}{*}{$P$-value } \\
\hline & CSMO & CSM20 & CSM40 & CSM60 & \\
\hline Met & $24.71 \pm 0.82$ & $27.18 \pm 1.02$ & $24.85 \pm 0.76$ & $25.44 \pm 0.41$ & 0.18 \\
\hline Phe & $22.61 \pm 1.06$ & $19.05 \pm 0.86$ & $21.52 \pm 0.97$ & $20.84 \pm 0.76$ & 0.12 \\
\hline Val & $136.59 \pm 4.51^{a}$ & $116.59 \pm 6.06^{a b}$ & $120.04 \pm 8.84^{a b}$ & $99.4 \pm 3.32^{b}$ & 0.02 \\
\hline lle & $90.87 \pm 3.94^{a}$ & $75.55 \pm 4.28^{a b}$ & $77.40 \pm 7.23^{a b}$ & $64.10 \pm 3.07^{b}$ & 0.03 \\
\hline Leu & $155.50 \pm 7.26^{a}$ & $127.01 \pm 6.96^{a b}$ & $132.74 \pm 13.41^{a b}$ & $113.02 \pm 4.23^{b}$ & 0.05 \\
\hline Thr & $44.63 \pm 2.01$ & $50.09 \pm 4.07$ & $54.56 \pm 2.47$ & $46.91 \pm 1.59$ & 0.12 \\
\hline Lys & $263.15 \pm 25.28$ & $222.67 \pm 14.77$ & $226.58 \pm 16.14$ & $210.76 \pm 12.62$ & 0.25 \\
\hline His & $10.46 \pm 0.22$ & $10.78 \pm 0.13$ & $10.26 \pm 0.25$ & $10.62 \pm 0.083$ & 0.31 \\
\hline Arg & $82.88 \pm 6.50^{\mathrm{a}}$ & $72.62 \pm 2.39^{a b}$ & $71.18 \pm 1.77^{a b}$ & $62.06 \pm 2.34^{b}$ & 0.03 \\
\hline EAA & $831.40 \pm 12.88^{a}$ & $721.53 \pm 37.34^{a b}$ & $755.09 \pm 19.84^{a b}$ & $644.21 \pm 26.79^{b}$ & 0.01 \\
\hline Glu & $44.70 \pm 5.24$ & $38.93 \pm 5.19$ & $37.47 \pm 4.53$ & $43.65 \pm 4.13$ & 0.67 \\
\hline Gly & $72.47 \pm 2.14$ & $69.60 \pm 1.95$ & $70.26 \pm 0.96$ & $75.77 \pm 0.45$ & 0.13 \\
\hline Ala & $88.71 \pm 3.68^{a}$ & $78.91 \pm 0.46^{b}$ & $88.19 \pm 0.54^{a b}$ & $95.37 \pm 2.02^{a}$ & 0.00 \\
\hline Cys & $1.83 \pm 0.56$ & $1.43 \pm 0.23$ & $1.54 \pm 0.45$ & $1.33 \pm 0.43$ & 0.87 \\
\hline Tyr & $18.66 \pm 0.39^{a}$ & $15.99 \pm 0.39^{b}$ & $19.36 \pm 0.59^{a}$ & $18.84 \pm 0.23^{a}$ & 0.00 \\
\hline Ser & $28.36 \pm 0.30$ & $27.85 \pm 0.75$ & $28.21 \pm 0.94$ & $31.16 \pm 1.29$ & 0.10 \\
\hline Tau & $75.98 \pm 2.17$ & $81.29 \pm 7.12$ & $75.53 \pm 0.62$ & $83.52 \pm 1.44$ & 0.41 \\
\hline NEAA & $318.46 \pm 9.97$ & $313.56 \pm 5.60$ & $320.56 \pm 5.08$ & $349.65 \pm 8.64$ & 0.06 \\
\hline TAA & $1136.02 \pm 51.75$ & $1035.00 \pm 31.76$ & $1075.00 \pm 18.25$ & $993.86 \pm 35.42$ & 0.13 \\
\hline
\end{tabular}

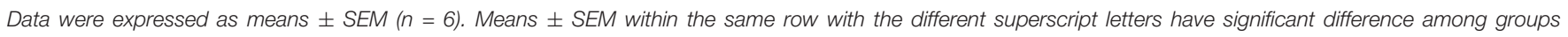
$(P<0.05)$.

EAA, total essential AA; NEAA, total non-essential AA; TAA, total AA.

EAAs and total EAA in plasma $(P>0.05)$. However, compared with the control diet, the CSM60 diet significantly decreased the concentrations of valine, isoleucine, leucine, arginine, and EAA in plasma $(P<0.05)$. Furthermore, the CSM40 and CSM60 diets also had no significant difference in changing the concentrations of alanine and tyrosine compared with the control diet $(P>0.05)$. No significant difference was detected in other AAs among the four different treatments $(P>0.05)$.

\section{Whole Body Fatty Acid Profile}

The changes in the whole body fatty acid profile after fish were fed with different diets were presented in Table 8. Compared with the control diet, the CSM20 diet had no significant difference in any of body fatty acid contents detected in the present study $(P>0.05)$. Compared with the fish fed with the CSM0 diets, fish fed with the 60\% CSM replacement diets exhibited notably reduced whole body C18:2 and $\Sigma$ n- 6 PUFA contents $(P<0.05)$. The CSM40 and CSM60 diets also significantly 
TABLE 8 | Whole body fatty acids content (\% total fatty acids).

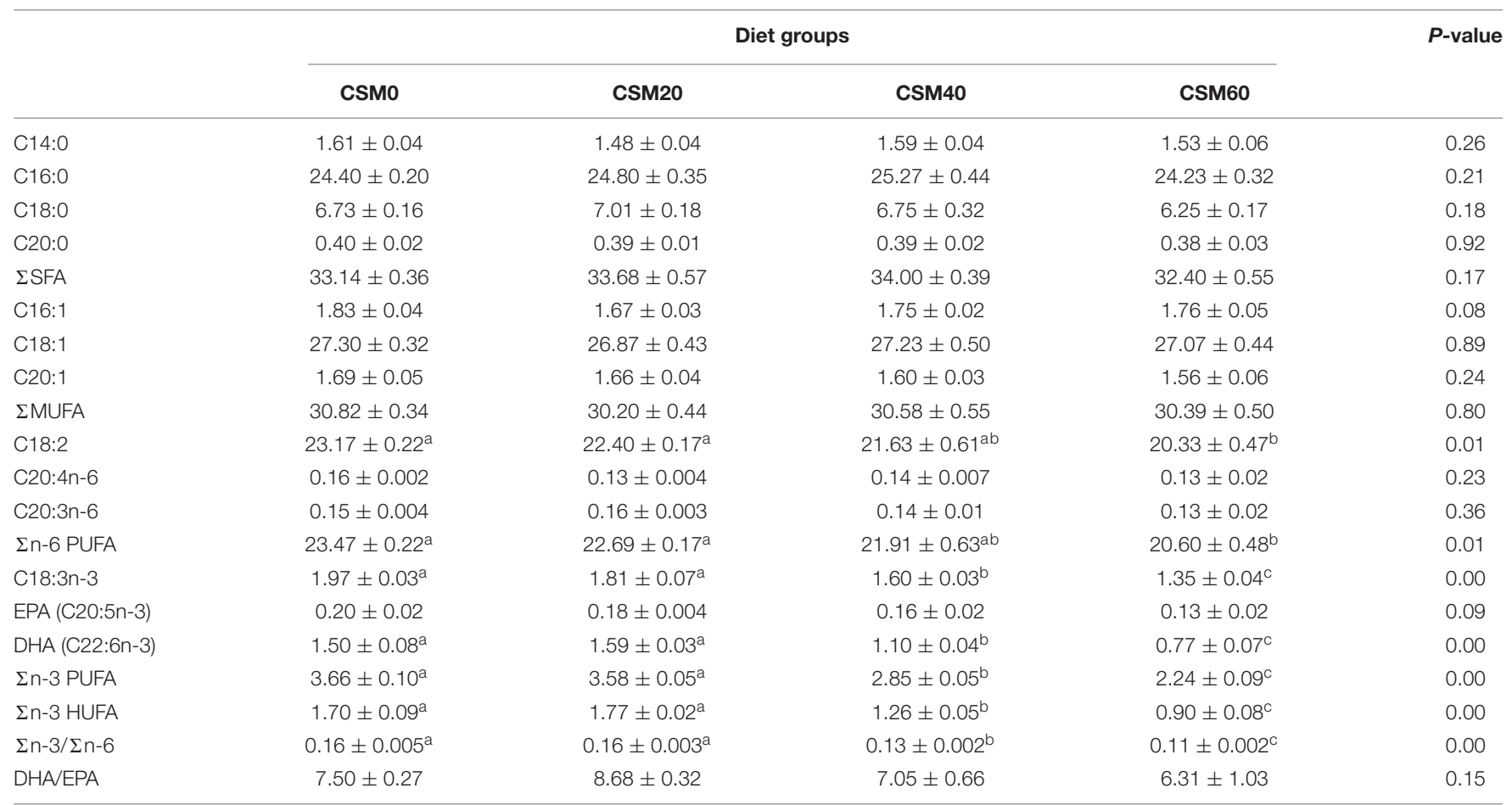

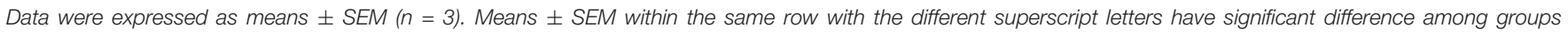
$(P<0.05)$.

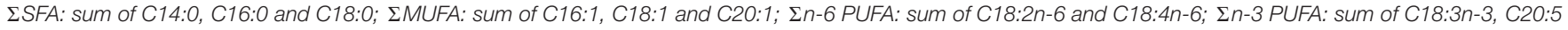
and C22:6. $\Sigma n-3 / \Sigma n-6: \Sigma n-3$ PUFA/ $\Sigma$ n-6 PUFA.

SFA, saturated fatty acids; MUFA, mono-unsaturated fatty acids; n-6 PUFA, $n$-6 poly-unsaturated fatty acids; $n$-3 PUFA, $n$-3 poly-unsaturated fatty acid.

decreased the whole body content of C18:3n-3, DHA (C22:6n3), $\Sigma$ n-3 PUFA, $\Sigma$ n-3 HUFA, and $\Sigma$ n- $3 / \Sigma$ n- 6 compared with the control diet $(P<0.05)$. No significant effect was observed in the whole body C20:4n-6, C20:3n-6, EPA, and DHA/EPA among the different CSM gradient replacement diets $(P>0.05)$. Furthermore, CSM gradient replacements also had no significant effect on altering the whole body contents of individual and total saturated fatty acids (SFA) and monounsaturated fatty acids (MUFA) $(P>0.05)$.

\section{Regulations of the Nutrient Sensing Signaling Pathways}

Gene and protein phosphorylation levels of the key regulators involved in nutrient sensing signaling pathways in the liver after fish were fed different diets are presented in Figure 1. Compared with golden pompano fed with CSM0 diet, golden pompano fed with the CSM20 diet did not change the mRNA expression levels of TOR, S6, and 4EBP1 $(P>0.05)$. The CSM60 diet significantly reduced the gene expression level of TOR and S6 compared with the control diet $(P<0.05)$. On the other hand, CSM substitution markedly promoted the gene expression of eIF $2 \alpha$ and REDD1 in the liver $(P<0.05)$. The CSM20 diet had no effect on altering the mRNA expression levels of ATF4 and CHOP compared with the control diet $(P>0.05)$. However, both the CSM40 and CSM60 diets increased the mRNA expression of ATF4 and CHOP in the liver $(P<0.05)$. Moreover, the CSM20 and CSM40 diets also had no effect in changing the protein phosphorylation levels of TOR and S6 compared with the CSM0 diet $(P>0.05)$. Conversely, the CSM60 diet decreased the protein phosphorylation level of $S 6$ and increased the protein phosphorylation level of eIF2 $\alpha$ than the control diet $(P<0.05)$.

\section{Gene Expression Pattern of the Lipid Metabolism}

The gene expression patterns of the key enzymes and regulators involved in lipid anabolism, catabolism, and transportation in the liver are shown in Figures 2A-C. Compared with the fish fed with CSM0 diets, the fish fed with CSM20 and CSM40 diets did not markedly decrease the gene expression of FAS, AGPAT3, SREBP1, and PPAR- $\gamma(P>0.05)$. In addition, the CSM20 diet also had no effect on reducing the mRNA expression of FAD, elovl5, and PPAR- $\alpha$ compared with the CSM0 $\operatorname{diet}(P>0.05)$. In contrast, the CSM60 diets markedly lowered the gene expression levels of FAS, ACC, AGPAT3, FAD, elovl5, SREBP1, PPAR- $\alpha$, and PPAR- $\gamma$ $(P<0.05)$. For lipid catabolism, no changes were detected between the CSM0 and CSM20 diets for the gene expression of the key enzymes involved in lipid catabolism $(P>0.05)$. The CSM60 diet significantly increased the mRNA expression levels of LPL and CPT1 compared with the other three diets $(P<0.05)$. The gene expression patterns of FABP1 and APROB100 showed a decreasing trend with an increasing 


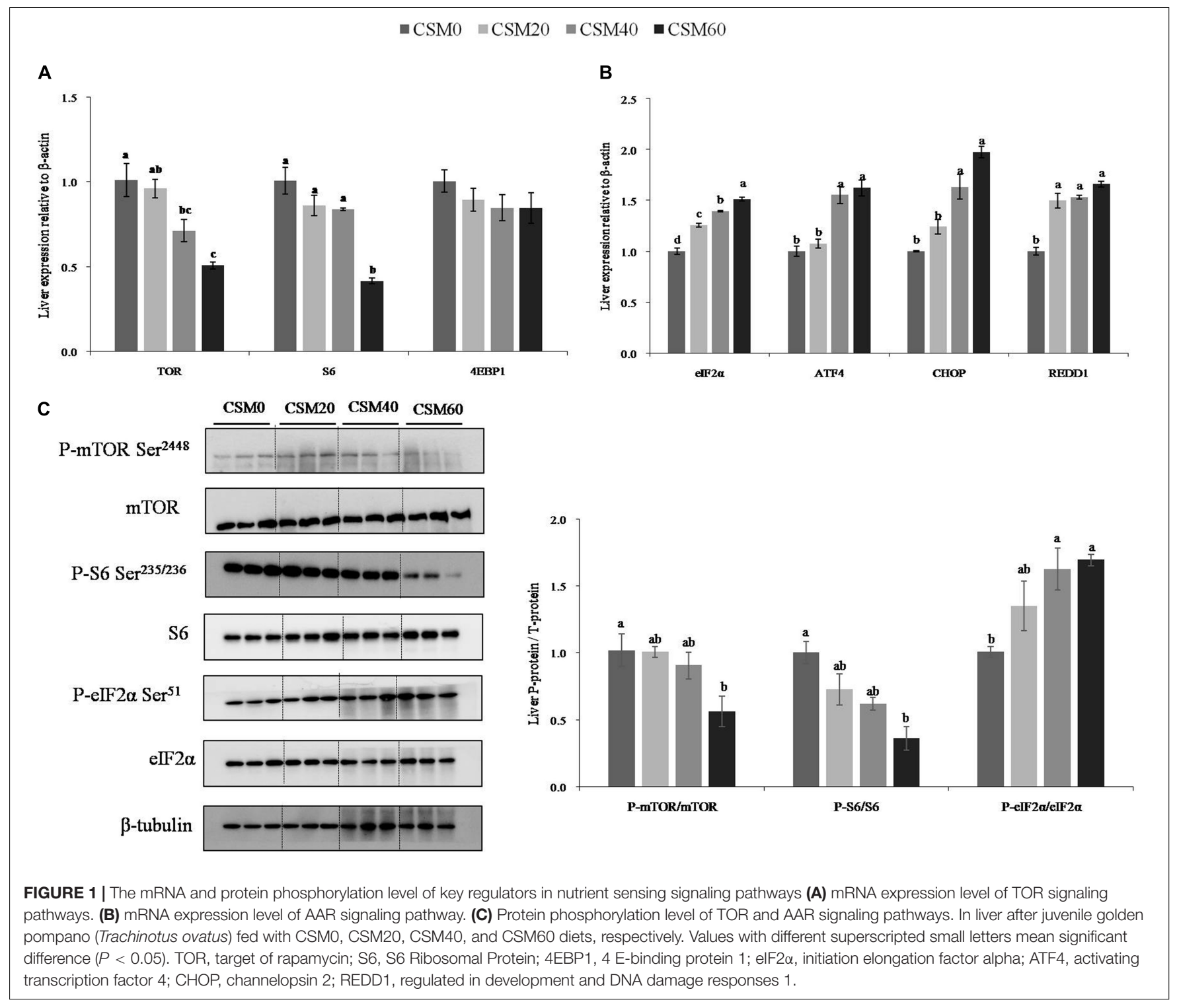

CSM replacement ratio. The fish fed with 60\% dietary CSM replacement diet showed significantly lower levels of FABP1 and APROB100 than the fish fed with the control diet $(P<0.05)$. However, the CSM20 diet did not change the gene expression of FABP1 and APROB100 compared with the control diet $(P>0.05)$.

\section{mRNA Expression Level of the Key Enzymes and Transporters in Glucose Metabolism}

As presented in Figure 3, the mRNA expression levels of the key enzymes and transporters in glucose metabolism showed a decreasing trend with increasing FM replacement. The CSM20 diet had no effect on inhibiting the mRNA expression levels of the g6pdh, pk, pepck, and glut4 compared with the control diet $(P>0.05)$. However, the CSM40 and CSM60 diets significantly decreased the mRNA expression levels of g6pdh, hk, pk, pfk-1, pepck, and glut2 $(P<0.05)$. Moreover, a marked decrease was observed in the gene expression of hk, pfk-1, and glut 2 when only $20 \%$ FM was replaced by CSM $(P<0.05)$.

\section{Cottonseed Meal Gradient Replacements Modulated the mRNA Expression Level of the Key Enzymes in Antioxidant Defense}

Figure 4 described the mRNA expression level changes of the key enzymes in antioxidant defense after golden pompano were fed CSM gradient replacement diets. Compared with the fish fed with the CSM0 diets, the fish fed with the CSM20 diet did not show marked effects in the mRNA expression levels of MnSOD, Nrf2, and Keap1 in the intestine or CAT in the kidney $(P>0.05)$. Nevertheless, the CSM40 and CSM60 diets showed higher gene expression levels of the MnSOD, CAT, and Nrf2 


\section{$\because \operatorname{CSM} 0 \quad \operatorname{CSM} 20=\operatorname{CSM} 40 \sim \operatorname{CSM} 60$}

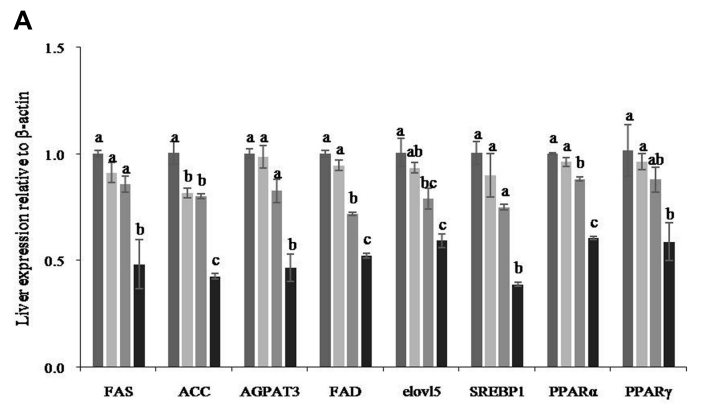

B

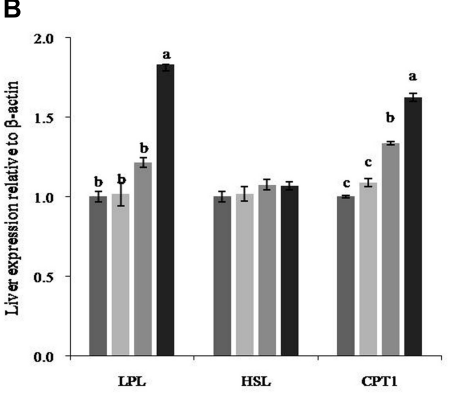

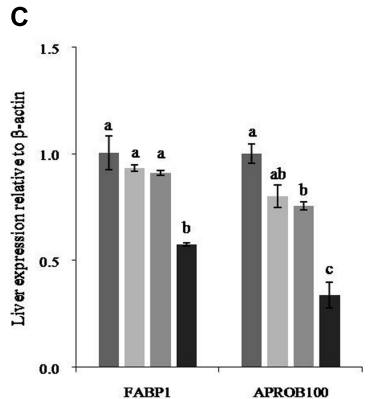

FIGURE 2 | Diagrammatic representation of the gene expression pattern of the key transcripts involved in lipid metabolism after juvenile golden pompano (Trachinotus ovatus) fed with CSM0, CSM20, CSM40, and CSM60 diets, respectively. (A) Lipid anabolism. (B) Lipid catamolism. (C) Lipid transporters. Values with different superscripted small letters mean significant difference $(P<0.05)$. FAS, fatty acid synthetase; ACC, acetyl-CoA carboxylase; AGPAT3, 1-acylglycerol-3-phosphate acyltransferase 3; FAD, fatty acyl desaturase; elovl5, elongase of very long-chain fatty acids 5; SREBP1, sterol regulatory element binding protein-1; PPAR $\alpha$, peroxisome proliferator activated receptors-alpha; PPAR $\gamma$, peroxisome proliferator-activated receptors gamma; LPL, lipoprotein lipase; HSL, hormone-sensitive lipase; CPT1, carnitine palmitoyl transferase 1; FABP1, fatty acid binding protein 1; APROB100, apolipoprotein b 100.

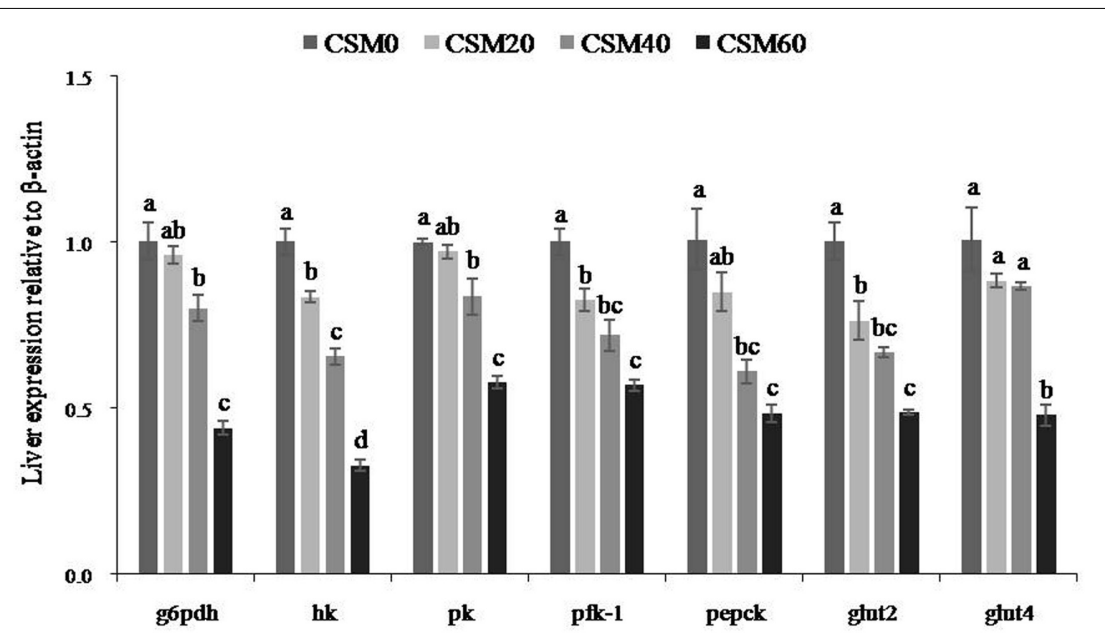

FIGURE 3 | RT-qPCR analysis of the key enzymes and transporters in glucose metabolism after juvenile golden pompano (Trachinotus ovatus) fed with CSM0, CSM20, CSM40, and CSM60 diets, respectively. Values with different superscripted small letters mean significant difference $(P<0.05)$. g6pdh, glucose-6-phosphate; hk, hexokinase; pk, pyruvate kinase; pfk-1, phospho fructokinase-1; pepck, phosphoenolpyruvate carboxykinase; glut2, glucose transport protein 2; glut4, glucose transport protein 4.

in the intestine, and MnSOD, CAT, and HO-1 in the kidney $(P<0.05)$. Moreover, the CSM60 diet presented lower Keap1 expression than the control diet in both the intestine and kidney $(P<0.05)$. CSM replacements also had no significant effect on changing the mRNA expression of $\mathrm{HO}-1$ in the intestine and Nrf2 in the kidney $(P>0.05)$.

\section{Cottonseed Meal Gradient Replacements Modified the Immune Response}

Immune-response-related gene mRNA expression in the intestine and kidney is displayed in Figure 5. Compared with the CSM0 diet, the CSM20 diet had no effect on changing the gene expression of IL-10, TGF $\beta$-1, C4, and Hsp90 in the intestine $(P>0.05)$. In addition, the CSM20 diet also did not alter the mRNA expression of IL-10, IL-8, TGF $\beta$-1, C4, C-lyz, and Hsp90 in the kidney compared with the CSM0 diet $(P>0.05)$. However, compared with the control diet, the CSM40 and CSM60 diets had a marked effect on increasing the gene expression of IL-10, IL-8, C4,Hsp70, and I $\kappa-\alpha$ in the intestine and IL-10, IL-8, C-lyz, and Hsp90 in the kidney $(P<0.05)$. On the other hand, CSM60 diets significantly inhibited the mRNA expression of TGF $\beta-1$ in both the intestine and the kidney compared with the control diet $(P<0.05)$. There was no significant difference in the expression of C-lyz in the intestine or Hsp70 and IкB- $\alpha$ in the kidney among the different groups $(P>0.05)$.

\section{DISCUSSION}

The present study showed that CSM replaced with 40\% FM protein did not decrease the FER and PER; however, based on the WGR and SGR, CSM could only substitute $20 \%$ of FM protein in 

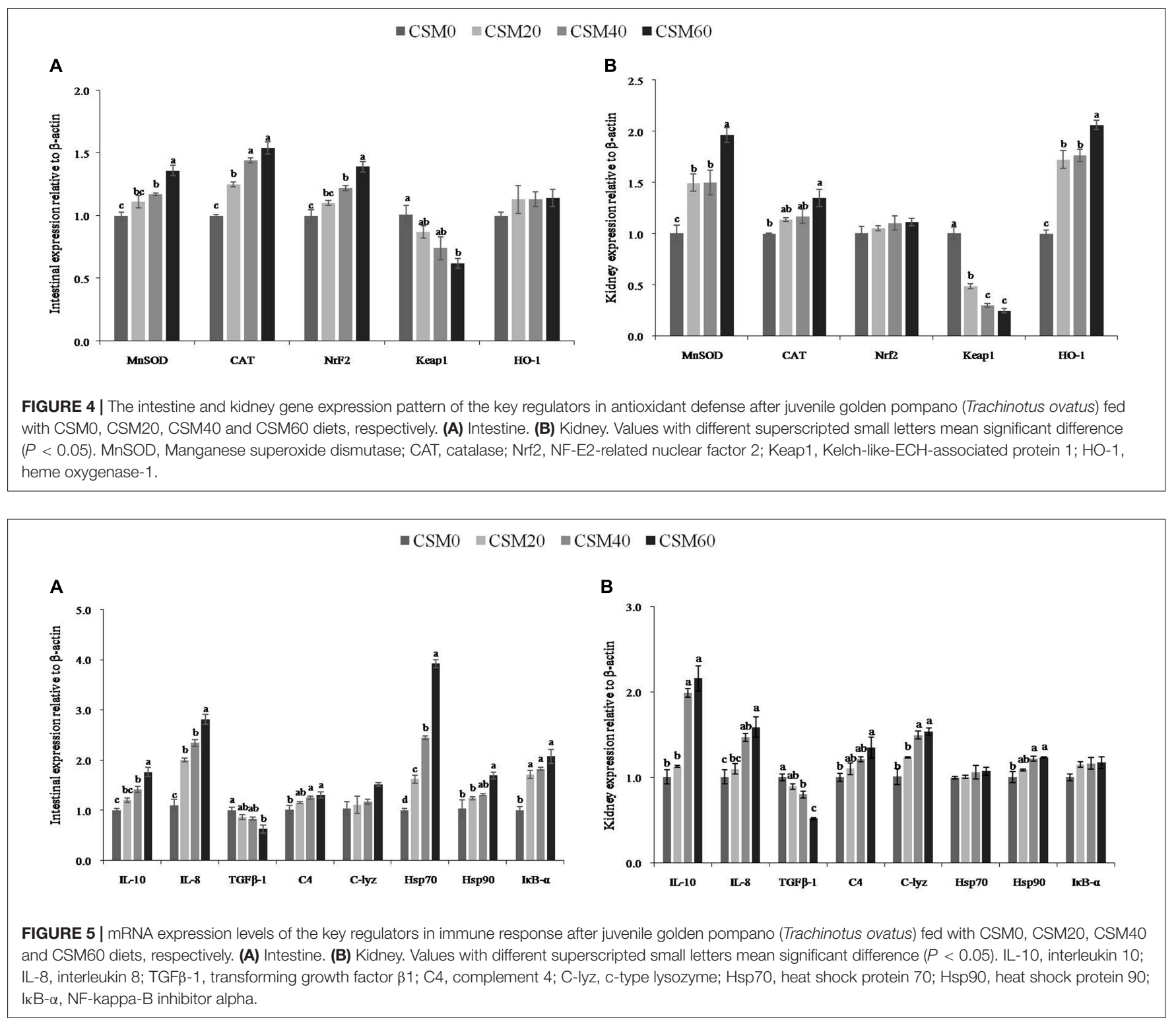

the golden pompano diet. Moreover, compared with the CSM0 diet, only the CSM20 diet did not decrease the dry matter and protein apparent digestibility compared with the control diet. However, higher CSM inclusion significantly decreased growth performance and feed utilization. All the results were consistent with previous studies on juvenile black sea bass Centropristis striata (Anderson et al., 2016), juvenile hybrid grouper (Ye et al., 2020), and crucian carp (Carassius auratus gibelio) (Gui et al., 2010). Golden pompano, a carnivorous fish species, has a limited ability to utilize dietary plant protein sources. Wu et al. (2014) reported that soy protein concentrate alone could substitute $20 \%$ FM without supplemental dietary taurine in golden pompano. Similarly, in a carnivorous fish, dietary replacement of over $25.3 \%$ FM with CSM could markedly inhibit the growth performance of Ussuri catfish (Bu et al., 2017). The formulation of the present diet was designed based on the commercial diet of golden pompano.
A variety of plant protein sources including corn gluten meal, soybean meal, and peanut meal were used as the main protein sources in the diet, and the supplemental amount of FM in the control group was only $25 \%$. Therefore, the replacement of $>20 \%$ FM with CSM inhibited growth performance.

Understanding the changes in physio-biochemical and molecular responses after fish were fed a CSM substitution diet is also an important way to improve the substitution efficiency (Dai et al., 2015; Xu et al., 2016). Plasma nutrient and free AA contents were intuitive indexes reflecting the situation of feed ingestion (Wang W.Q. et al., 2021; Wang Z. et al., 2021). In the present study, CSM replacement had a profound effect on altering the plasma biochemical index. CSM substitution at $40 \%$ and $60 \%$ markedly decreased the total TP, TG, and HLD, which was consistent with a study on largemouth bass and turbot (Wang et al., 2020). The plasma-free AA concentration was 
considered the main driving force determining the metabolic rate (Liao et al., 2015; Wei et al., 2020). Higher plasma-free AA concentrations provided abundant substrates that could improve protein anabolism. Previous studies using Antarctic krill as a FM substitution in large yellow croaker and poultry by-product meal as a FM substitution in largemouth bass showed lower levels of individual AAs after FM was substituted by other protein sources (Wei et al., 2019; Wang et al., 2020). This study also presented a declining trend of plasma individuals and total EAA after CSM replaced $60 \%$ of FM in the diet. Compared with NEAA, the concentrations of some EAAs were more sensitive to dietary composition. Lower plasma nutrient and free AA concentrations decreased the metabolic rate of nutrients and further limited the deposition of body nutrients.

The fatty acid content profile of the whole body is another important indicator to reflecting the optimal substitution ratio of alternative protein sources (Hill et al., 2019). From a human nutritive perspective, fish with a high n-3 PUFA conent are regarded as more valuable than those with a high n-6 PUFA content (Simopoulos, 2002). The present study demonstrated that the inclusion of CSM in the diet had no significant effect on regulating the content of SFA and MUFA. In contrast, the CSM 40 and CSM60 replacement diets decreased the contents of DHA, $\Sigma$ n-3 PUFA, $\Sigma$ n-3 HUFA, and $\Sigma$ n-3/ $\Sigma$ n-6 compared with the control diet. Moreover, decreased profiles of whole body C18:2, $\Sigma$ n-6 PUFA, and C18:3n-3 were observed only when CSM was replaced at a higher ratio (CSM60 diet). A similar suppression of whole body $\Sigma$ n-3 PUFA and $\Sigma$ n-3 HUFA was observed in a study using a poultry by-product meal ingredient replacement diet in the hybrid grouper (Wang Z. et al., 2021). The results of whole body fatty acid contents indicated that CSM could substitute 20-40\% FM without affecting the fatty acid compositions of golden pompano.

To illustrate the underlying mechanisms of the changing growth performance and nutrient metabolism after CSM replaced FM, the present study also focused on the molecular response of nutrient deposition and metabolism. TOR and AAR are two complementary signaling pathways that respond to dietary nutrient levels (Kimball, 2007; Hietakangas and Cohen, 2009). The two signaling pathways were more sensitive to the dietary AA profile (Gallinetti et al., 2013). An unbalanced AA profile was the first limiting factor restricting the utilization of alternative protein sources in aquafeeds ( $\mathrm{Li}$ et al., 2009). Our previous study on turbot and largemouth bass demonstrated that FM replaced by other protein sources with or without AA supplementation significantly varied the mRNA and protein expression levels of the key regulators in the TOR and AAR signaling pathways (Song et al., 2016; Wang W.Q. et al., 2021). Consistent with our previous studies, the present study on golden pompano also revealed a lower expression level of the key regulators involved in TOR signaling and a higher expression level of the key regulators involved in AAR signaling at both the mRNA and protein phosphorylation levels after FM was replaced by CSM at $40-60 \%$. The liver is the main organ that regulates metabolism overall. The TOR signaling pathway is the regulating center for nutrient metabolism (Hayashi and Proud, 2007; Irm et al., 2020). Therefore, on further exploring the mRNA regulation of glucose and lipid metabolism, we found that higher CSM replacement significantly inhibited the gene expression level of the key enzymes and regulators in lipid anabolism and lipid transporters, and elevated the gene expression level of the key enzymes in lipid catabolism. Similarly, CSM substitution also suppressed the mRNA expression level of the key enzymes and transporters involved in glucose metabolism. Inhibited nutrient anabolism and activated nutrient catabolism may provide a reasonable explanation for the reduced growth performance and nutrient metabolism after FM was replaced by CSM.

In addition to affecting nutrient metabolism, alternative protein source-substituted FM also has a profound effect on health status by regulating antioxidant defense and immune response (He et al., 2021). AST, ALT, ALP, and LDH are important indicators that reflect liver function (Hyder et al., 2013). The increasing activities of these enzymes in plasma and liver imply liver damage (Regmi et al., 2017). Bu et al. (2017) reported that the activities of plasma ALT and LDH significantly increased when the CSM substitution level was $>40 \%$ in Ussuri catfish. The present study also found that the activities of AST, ALT, ALP, LDH, and CK in the plasma and liver of juvenile golden pompano that was fed a large proportion of CSM inclusion diets (CSM40 and CSM60 diets) were higher than those in fish fed the CSM0 diet. These results confirmed liver damage when a higher ratio of CSM was supplemented in the diet of golden pompano. Moreover, the intestine and kidney are considered to be the key organ responses for the body's immune status (Awad et al., 2015; Lauriano et al., 2016; Wu et al., 2017). The intestine is not only considered the main site of the response to nutrient digestion and absorption but also the largest part of the immune system, which contains many lymphoid cells, macrophages, eosinophils, and neutrophilic granulocytes (Rombout et al., 2011). The present study was conducted to understand the overall immune response that focused on both the intestine and the kidney. The immune status was closely associated with inflammation which was mainly regulated by inflammatory cytokines. TNF, IL, and TGF are cytokines that play a vital role in body immunity (Delcenserie et al., 2008). In this study, a high level of CSM (CSM60 diet) in the diet markedly elevated the mRNA expression of IL$10, \mathrm{IL}-8, \mathrm{C} 4, \mathrm{Hsp} 70$, and IкB- $\alpha$ in the intestine and kidney. Moreover, data on the gene expression level in the intestine and kidney also demonstrated that CSM replacement activated the antioxidant defense. These results suggested that proportionate CSM substitution (20-40\%) had no effects on activating the immune response and antioxidant defense. However, higher CSM substitution induced the immune response and antioxidant defense and further damaged the health status. Overall, based on the immune response, CSM could substitute $20-40 \%$ FM in the present diet formulation.

\section{CONCLUSION}

Based on the results, the present study demonstrated that CSM could successfully substitute $20 \%$ dietary FM in the diet of the golden pompano with an initial body weight of approximately 28.4 g. Using CSM as a substitute for FM has promising prospects 
in golden pompano diets. However, higher CSM inclusion could affect growth performance by regulating TOR and AAR signaling and further modifying the nutrient metabolism. Additionally, higher CSM replacement could also alter the fish health status by regulating the immune response and antioxidant defense. This study proposes the optimal inclusion level of CSM in the diet and also contribute to understanding the mechanisms of dietary CSM in growth performance, nutrient metabolism, and immune response in golden pompano.

\section{DATA AVAILABILITY STATEMENT}

The original contributions presented in the study are included in the article/Supplementary Material, further inquiries can be directed to the corresponding author/s.

\section{ETHICS STATEMENT}

The animal study was reviewed and approved by the University Animal Care and Use Committee of the South China Normal University (an approval reference number 1002019-02-0016).

\section{REFERENCES}

Anderson, A. D., Alam, M. S., Watanabe, W. O., Carroll, P. M., Wedegaertner, T. C., and Dowd, M. K. (2016). Full replacement of menhaden fish meal protein by low-gossypol cottonseed flour protein in the diet of juvenile black sea bass Centropristis striata. Aquaculture. 464, 618-628. doi: 10.1016/j.aquaculture. 2016.08.006

Awad, E., Awaad, A. S., and Esteban, M. A. (2015). Effects of dihydroquercetin obtained from deodar (Cedrus deodara) on immune status of gilthead seabream (Sparus aurata L.). Fish Shellfish Immunol. 43, 43-50. doi: 10.1016/j.fsi.2014.12. 009

Bian, F. Y., Zhou, H. H., He, G., Wang, C., Peng, H., Pu, X., et al. (2017). Effects of replacing fishmeal with different cottonseed meals on growth, feed utilization, haematological indexes, intestinal and liver morphology of juvenile turbot (Scophthalmus maximus L.) Aquacult. Nutr. 23, 1429-1439. doi: 10.1111/anu. 12518

Bu, X. Y., Chen, A. J., Lian, X. Q., Chen, F. Y., Zhang, Y., Muhammad, I., et al. (2017). An evaluation of replacing fish meal with cottonseed meal in the diet of juvenile ussuri catfish Pseudobagrus ussuriensis: growth, antioxidant capacity, nonspecific immunity and resistance to Aeromonas hydrophila. Aquaculture. 479, 829-837. doi: 10.1016/j.aquaculture.2017.07.032

Cai, L. S., Wang, L., Song, K., Lu, K. L., Zhang, C. X., and Rahimnejad, S. (2020). Evaluation of protein requirement of spotted seabass (Lateolabrax maculatus) under two temperatures, and the liver transcriptome response to thermal stress. Aquaculture 516, 734615. doi: 10.1016/j.aquaculture.2019.734615

Dai, W. W., Panserat, S., Plagnes-Juan, E., Seiliez, I., and Skiba-Cassy, S. (2015). Amino acidsattenuate insulin action on gluconeogenesis and promote fattyacid biosynthesis via mTORC1 signaling pathway in trouthepatocytes. Cell. Physiol. Biochem. 36, 1084-1100. doi: 10.1159/000430281

Delcenserie, V., Martel, D., Lamoureux, M., Amiot, J., Boutin, Y., and Roy, D. (2008). Immunomodulatory effects of probiotics in the intestinal tract. Curr. Issues Mol. Biol. 10, 37-54. doi: 10.21775/cimb.010.037

Dossou, S., Koshio, S., Ishikawa, M., Yokoyama, S., Dawood, M. A. O., Basuini, M. F. E., et al. (2018). ). Effect of partial replacement of fish meal by fermented rapeseed meal on growth, immune response and oxidative condition of red sea bream juvenile, Pagrus major. Aquaculture 490, 228-235.

Gallinetti, J., Harputlugil, E., and Mitchell, J. R. (2013). Amino acid sensing in dietary restriction-mediated longevity: roles of signal-transducing

\section{AUTHOR CONTRIBUTIONS}

FS and KM designed the study. YQ and $\mathrm{CH}$ performed the study. PY and WW analyzed the data. FS, JW, and QQ revised the manuscript. All authors read and approved the final manuscript.

\section{FUNDING}

This work was supported by the Fund of Southern Marine Science and Engineering Guangdong Laboratory (Zhanjiang) (ZJW2019-06), the Foundation and Applied Basic Research Fund Project of Guangdong Province (2019A1515110606), the State Key Laboratory of Developmental Biology of Freshwater Fish and Research (201KF007), and the Research and Development Fund for Young Teachers of South China Normal University (19KJ03).

\section{SUPPLEMENTARY MATERIAL}

The Supplementary Material for this article can be found online at: https://www.frontiersin.org/articles/10.3389/fmars. 2021.775575/full\#supplementary-material

kinases GCN2 and TOR. Biochem. J. 449, 1-10. doi: 10.1042/BJ201 21098

Gatlin, D. M., Barrows, F. T., Brown, P., Dabrowski, K., Gaylord, T. G., Hardy, R. W., et al. (2007). Expanding the utilization of sustainable plant products in aquafeeds: a review. Aquac. Res. 38, 551-579. doi: 10.1111/j.1365-2109.2007. 01704.x

Gui, D., Liu, W. B., Shao, X. P., and Xu, W. N. (2010). Effects of different dietary levels of cottonseed meal protein hydrolysate on growth, digestibility, body composition and serum biochemical indices in crucian carp (Carassius auratus gibelio). Anim. Feed Sci. Technol 156, 112-120. doi: 10.1016/j.anifeedsci.2010. 01.012

Hayashi, A. A., and Proud, C. G. (2007). The rapid activation of protein synthesis by growth hormone requires signaling through mTOR. Am. J. Physiol. Endocrinol. Metab. 292, 1647-1655. doi: 10.1152/ajpendo.00674.2006

He, Y. F., Guo, X. W., Tan, B. P., Dong, X. H., Yang, Q. H., Liu, H. Y., et al. (2021). Partial fishmeal protein replacement with peptides from swine blood modulates the nutritional status, immune response, and intestinal microbiota of hybrid groupers (female Epinephelus fuscoguttatus $\times$ male E.lanceolatus). Aquaculture 533, 736154. doi: 10.1016/j.aquaculture.2020.736154

Hietakangas, V., and Cohen, S. M. (2009). Regulation of tissue growth through nutrient sensing. Annu. Rev. Genet. 43, 389-410. doi: 10.1146/annurev-genet102108-134815

Hill, J. C., Alam, M. S., Watanabe, W. O., Carroll, P. M., Seaton, P. J., and Bourdelais, A. J. (2019). Replacement of menhaden fish mealby poultry byproduct meal in the diet of juvenile red porgy. N. Am. J. Aquac. 81, 81-93. doi: 10.1002/naaq.10074

Hu, C. L., Li, X. H., He, X. Y., Yuan, X. L., Wang, R. X., Han, J., et al. (2021). Industry development report on cotton processing industry 2020. Cotton Processing in China. 2, 4-15.

Hyder, M. A., Hasan, M., and Mohieldein, A. (2013). Comparative levels of ALT, AST, ALPand GGT in liver associated diseases. Eur. J. Exp. Biol. 3, 280-284.

Irm, M., Taj, S., Jin, M., Andriamialinirina, H. J. T., Cheng, X., and Zhou, Q. C. (2020). Influence of dietary replacement of fish meal with fish soluble meal on growth and TOR signaling pathway in juvenile black sea bream (Acanthopagrus schlegelii). Fish Shellfish Immunol. 101, 269-276. doi: 10.1016/j.fsi.2020. 03.053

Kimball, S. R. (2007). The role of nutrition in stimulating muscle protein accretion at the molecular level. Biochem.Soc. Trans 35, 1298-1301. 
Lauriano, E. R., Pergolizzi, S., Capillo, G., Kuciel, M., Alesci, A., and Faggio, C. (2016). Immunohistochemical characterization of Toll-like receptor 2 in gut epithelial cells and macrophages of goldfish Carassius auratus fed with a highcholesteroldiet. Fish Shellfish Immunol. 59, 250-255. doi: 10.1016/j.fsi.2016.11. 003

Li, M. H., and Robinson, E. H. (2006). Use of cottonseed meal in aquatic animal diets: a review. N. Am. J. Aquac. 68, 14-22. doi: 10.1577/A05-028.1

Li, P., Mai, K. S., Trushenski, J., and Wu, G. Y. (2009). New developments in fish amino acid nutrition: towards functional and environmentally oriented aquafeeds. Amino Acids. 37, 43-53. doi: 10.1007/s00726-008-0171-1

Liao, S. F., Wang, T. J., and Regmi, N. (2015). Lysine nutrition in swine and the related monogastric animals: muscle protein biosynthesis and beyond. Springer Plus. 4, 147-158. doi: 10.1186/s40064-015-0927-5

Lim, S. J., and Lee, K. J. (2009). Partial replacement of fish meal by cottonseed meal and soy-bean meal with iron and phytase supplementation for parrot fish Oplegnathus fasciatus. Aquaculture. 290, 283-289. doi: 10.1016/j.aquaculture. 2009.02.018

Lu, F., Haga, Y., and Satoh, S. (2015). Effects of replacing fish meal with rendered animal protein and plant protein sources on growth response, biological indices, and amino acid availability for rainbow trout Oncorhynchus mykiss. Fish. Sci. 81, 95-105. doi: 10.1007/s12562-014-0818-7

Ma, X. Z., Wang, F., Han, H., Wang, Y., and Lin, Y. Y. (2014). Replacement of dietary fish meal with poultry by-product meal and soybean meal for golden pompano, Trachinotus ovatus, reared in net pens. J. World Aquacult. Soc. 45, 662-671. doi: 10.1111/jwas.12154

Mourente, G., Tocher, D. R., Diaz-Salvago, E., Grau, A., and Pastor, E. (1999). Study of the n-3 highly unsaturated fatty acids requirement and antioxidant status of Dentex dentexlarvae at the Artemia feeding stage. Aquaculture 179, 291-307. doi: 10.1016/S0044-8486(99)00166-0

Regmi, N., Wang, T., Crenshaw, M. A., Rude, B. J., and Liao, S. F. (2017). Effects of dietary lysinelevels on the concentrations of selected nutrient metabolites in blood plasma of late-stage finishing pigs. J. Anim. Physiol. Anim. Nutr. 102, 403-409. doi: 10.1111/jpn.12714

Rombout, J. H. W. M., Abelli, L., Picchietti, S., Scapigliati, G., and Kiron, V. (2011). Teleost intestinal immunology. Fish Shellfish Immunol 31, 616-626.

Simopoulos, A. P. (2002). The importance of the ratio of omega-6/omega-3 essential fattyacids. Biomed. Pharmacother 56, 365-379.

Song, F., Xu, D. D., Mai, K. S., Zhou, H. H., Xu, W., and He, G. (2016). Comparative study on the cellular and systemic nutrient sensing and intermediary metabolism after partial replacement of fishmeal by meat and bone meal in the diet of Turbot (Scophthalmus maximus L.). PLoS One. 11:e0165708. doi: 10.1371/journal.pone.0165708

Song, Z. D., Li, H. Y., Wang, J. Y., Li, P. Y., Sun, Y. Z., and Zhang, L. M. (2014). Effects of fishmeal replacement with soy protein hydrolysates on growth performance, blood biochemistry, gastrointestinal digestion and muscle composition of juvenile starry flounder (Platichthys stellatus). Aquaculture 426-427, 96-104.

Sullivan, J. A., and Reigh, R. C. (1995). Apparent digestibility of selected feedstuffs in diets for hybrid striped bass (Morone saxatilis $q \mathrm{x}$ Morone chrysops $\left.\sigma^{7}\right)$. Aquaculture. 138, 313-322. doi: 10.1016/0044-8486(95)0 1071-8

Tan, X. H., Lin, H. Z., Huang, Z., Zhou, C. P., Wang, A. L., Qi, C. L., et al. (2016). Effects of dietary leucine on growth performance,feed utilization, nonspecific immune responses and gut morphology of juvenilegolden pompano Trachinotus ovatus. Aquaculture. 465, 100-107. doi: 10.1016/j.aquaculture. 2016.08.034

Tang, Y. Y., Zhang, J. N., Ai, C. X., and Hu, B. (2013). Review of nutrient requirements and formuladietary for Trachinotus ovatus. Feed Indust. 34, 4650 .

Wacyk, J., Powell, M., Rodnick, K., Overturf, K., Hill, R. A., and Hardy, R. (2012). Dietary protein source significantly alters growth performance, plasma variables and hepatic gene expression in rainbow trout (Oncorhynchus mykiss) fed amino acid balanced diets. Aquaculture. 35, 223-234. doi: 10.1016/j.aquaculture.2012. 05.013

Wan, M. G., Yin, P., Fang, W. P., Xie, S. W., Chen, S. J., Tian, L. X., et al. (2018). The effect of replacement of fishmeal by concentrated dephenolization cottonseed protein on the growth, body composition, haemolymph indexes and haematological enzyme activities of the Pacific white shrimp (Litopenaeus vannamei). Aquac. Nutr. 24, 1845-1854. doi: 10.1111/anu.12823

Wang, Q., He, G., Mai, K. S., Xu, W., and Zhou, H. H. (2015). Fishmeal replacement by mixed plantproteins and maggot meal on growth performance, target of rapamycin signalling andmetabolism in juvenile turbot (Scophthalmus maximus L.). Aquac. Nutr. 22, 752-758. doi: 10.1111/anu.12296

Wang, W. Q., Xu, Y. J., Chi, S. Y., Yang, P., Mai, K. S., and Song, F. (2020). Dietary lysine regulates bodygrowth performance via the Nutrient-sensing signaling pathways in largemouth bass (Micropterus salmoides). Front. Mar. Sci. 7:595682.

Wang, W. Q., Yang, P., He, C. Q., Chi, S. Y., Li, S. L., Mai, K. S., et al. (2021). Effects of dietary methionine on growth performance and metabolism through modulating nutrient-related pathways in largemouth bass (Micropterus salmoides). Aquacult. Rep. 20, 100642. doi: 10.1016/j.aqrep.2021.1 00642

Wang, Z., Qian, X. Q., Xie, S. Q., and Yun, B. (2021). Changes of growth performance and plasma biochemical parameters of hybrid grouper (Epinephelus lanceolatus $0^{7} \times$ Epinephelus fuscoguttatus ㅇ) in response to substitution of dietary fishmeal with poultry by-product meal. Aquacult. Rep. 18, 100516. doi: 10.1016/j.aqrep.2020.100516

Wei, Y. L., Li, B. X., Xu, H. G., and Liang, M. Q. (2020). Effects of lysine and leucine in free and differentdipeptide forms on the growth, amino acid profile and transcription of intestinalpeptide, and amino acid transporters in turbot (Scophthalmus maximus). Fish Physiol.Biochem. 46, 1795-1807. doi: 10.1007/ s10695-020-00828-2

Wei, Y. T., Shen, H. H., Xu, W. Q., Pan, Y., Chen, J., Zhang, W. B., et al. (2019). Replacement of dietary fishmeal by antarctic krill meal on growth performance, intestinal morphology, body composition and organoleptic quality of large yellow croaker Larimichthys crocea. Aquaculture 512, 734281.

Wu, P., Liu, Y., Jiang, W. D., Jiang, J., Zhang, Y. A., Zhou, X. Q., et al. (2017). Intestinal immune responses of jian carp against Aeromonas hydrophila depressed by choline deficiency: varied change patterns of mRNA levels of cytokines, tight junction proteins and related signaling molecules among three intestinal segments. Fish Shellfish Immunol. 65, 34-41. doi: 10.1016/j.fsi.2017. 03.053

Wu, Y., Han, H., Qin, J., and Wang, Y. (2014). Replacement of fishmeal by soy protein concentrate with taurine supplementation in diets for golden pompano (Trachinotus ovatus). Aquac. Nutr. 21, 214-222. doi: 10.1111/anu.12161

Xie, S. W., Wei, D., Yin, P., Zheng, L., Guo, T. Y., Liu, Y. J., et al. (2019). Dietary replacement of fish-meal impaired protein synthesis and immune response of juvenile pacific white shrimp, Litopenaeus vannamei at low salinity. Comp. Biochem. Physiol. B. Biochem. Mol. Biol. 228, 26-33. doi: 10.1016/j.cbpb.2018. 11.002

Xu, D. D., He, G., Mai, K. S., Zhou, H. H., Xu, W., and Song, F. (2016). Postprandial nutrient-sensing and metabolic responsesafter partial dietary fishmeal replacement by soybean meal in turbot (Scophthalmus maximus L.). Br. J.Nutr. 115, 379-388. doi: 10.1017/S0007114515004535

Xu, H. G., Ai, Q. H., Mai, K. S., Xu, W., Wang, J., Ma, H. M., et al. (2010). Effects of dietary arachidonic acid on growth performance, survival, immune response and tissue fatty acid composition of juvenile Japanese seabass, Lateolabrax japonicus. Aquaculture. 307, 75-82. doi: 10.1016/j.aquaculture.2010. 07.001

Xun, P. W., Lin, H. Z., Wang, R. X., Huang, Z., Zhou, C. P., Yu, W., et al. (2019). Effects of dietary vitamin B1 on growth performance, intestinal digestion and absorption, intestinal microflora and immune response of juvenile golden pompano (Trachinotus ovatus). Aquaculture. 506, 75-83. doi: 10.1016/j.aquaculture.2019.03.017

Yang, P., Wang, W. Q., Chi, S. Y., Mai, K. S., Song, F., and Wang, L. (2020). Effects of dietary lysine on regulating GH-IGF system, intermediate metabolism and immune response in largemouth bass (Micropterus salmoides). Aquacult. Rep. 17, 100323. doi: 10.1016/j.aqrep.2020.100323

Ye, G. L., Dong, X. H., Yang, Q. H., Chi, S. Y., Liu, H. Y., Zhang, H. T., et al. (2020). Low-gossypol cottonseed protein concentrate used as a replacement of fish meal for juvenile hybrid grouper (Epinephelus fuscoguttatus $q \times$ Epinephelus lanceolatus $\left.\sigma^{7}\right)$ : Effects on growth performance, immune responses and intestinal microbiota - science dire. Aquaculture 524, 735309. doi: 10.1016/j. aquaculture.2020 735309 
Zheng, Q. M., Wen, X. B., Han, C. Y., Li, H. B., and Xie, X. H. (2012). Effect of replacing soybean meal withcottonseed meal on growth, hematology, antioxidant enzymes activity and expressionfor juvenile grass carp, Ctenopharyngodon idellus. Fish Physiol. Biochem. 38, 1059-1069. doi: 10.1007/ s10695-011-9590-0

Zhou, W., Rahimnejad, S., Lu, K., Wang, L., and Liu, W. (2019). Effects of berberine on growth, liver histology, and expression of lipid-related genes in blunt snout bream (Megalobrama amblycephala) fed high-fat diets. Fish Physiol Biochem 45, 83-91.

Conflict of Interest: The authors declare that the research was conducted in the absence of any commercial or financial relationships that could be construed as a potential conflict of interest.
Publisher's Note: All claims expressed in this article are solely those of the authors and do not necessarily represent those of their affiliated organizations, or those of the publisher, the editors and the reviewers. Any product that may be evaluated in this article, or claim that may be made by its manufacturer, is not guaranteed or endorsed by the publisher.

Copyright (c) 2021 Qin, He, Wang, Yang, Wang, Qin, Mai and Song. This is an open-access article distributed under the terms of the Creative Commons Attribution License (CC BY). The use, distribution or reproduction in other forums is permitted, provided the original author(s) and the copyright owner(s) are credited and that the original publication in this journal is cited, in accordance with accepted academic practice. No use, distribution or reproduction is permitted which does not comply with these terms. 